Draft version March 20, 2018

Preprint typeset using IATEX style emulateapj v. 12/16/11

\title{
THE ROUTE TO MASSIVE BLACK HOLE FORMATION VIA MERGER-DRIVEN DIRECT COLLAPSE: A REVIEW
}

\author{
LuCIO MAYER
}

Center for Theoretical Astrophysics and Cosmology, Institute for Computational Science, University of Zurich, Winterthurerstrasse 190 , CH-8057 Zurich, Switzerland, and Kavli Institute for Theoretical Physics, Kohn Hall, University of California, Santa Barbara, CA 93106, USA, lmayer@physik.uzh.ch

Silvia BONOLI

Centro de Estudios de Fisica del Cosmos de Aragon, Plaza San Juan 1, Planta-2, E-44001, Teruel, Spain Draft version March 20, 2018

\begin{abstract}
The direct collapse model for the formation of massive black holes has gained increased support as it provides a natural explanation for the appearance of bright quasars already less than a billion years from the Big Bang. In this paper we review a recent scenario for direct collapse that relies on multiscale gas inflows initiated by the major merger of massive gas-rich galaxies at $z>6$, where gas has already achieved solar composition. Hydrodynamical simulations undertaken to explore our scenario show that supermassive, gravitationally bound compact gaseous disks weighing a billion solar masses, only a few pc in size, form in the nuclei of merger remnants in less than $10^{5} \mathrm{yr}$. These could later produce a supermassive protostar or supermassive star at their center via various mechanisms. Moreover, we present a new analytical model, based on angular momentum transport in mass-loaded gravitoturbulent disks. This naturally predicts that a nuclear disk accreting at rates exceeding $1000 M_{\odot} / \mathrm{yr}$, as seen in the simulations, is stable against fragmentation irrespective of its metallicity. This is at variance with conventional direct collapse scenarios, which require the suppression of gas cooling in metal-free protogalaxies for gas collapse to take place. Such high accretion rates reflect the high free-fall velocities in massive halos appearing only at $z<10$, and occur naturally as a result of the efficient angular momentum loss provided by the merger dynamics. We discuss the implications of our scenario on the observed population of high-z quasars and on its evolution to lower redshifts using a semi-analytical galaxy formation model. Finally, we consider the intriguing possibility that the secondary gas inflows in the unstable disks might drive gas to collapse into a supermassive black hole directly via the General Relativistic radial instability. Such dark collapse route could generate gravitational wave emission detectable via the future Laser Interferometer Space Antenna (LISA).
\end{abstract}

Keywords: galaxies: star formation; black holes; gravity

\section{ASTROPHYSICAL SCENARIOS FOR MASSIVE BH FORMATION AND THEIR AND CHALLENGES}

The first evidence on the existence of supermassive black holes, with masses in the range $10^{6}-10^{10} M_{\odot}$, comes from the discovery of quasars and radio galaxies in the 60s (Schmidt 1963). When X-ray satellites became available, many of these sources revealed also powerful X-ray emission (see, e.g., the review of Brandt \& Hasinger 2005). Galaxies presenting this kind of activity are generically referred to as Active Galactic Nuclei (AGNs), as the activity originates from the nuclear region of the galaxy itself. It was soon realized that the most natural way to explain the gargantuan luminosities associated with such astrophysical phenomena, which can outshine the galaxies that host them, is the energy radiation derived from the loss of potential energy of matter as it falls onto a massive compact object located in the galactic nucleus (Salpeter 1964; LyndenBell 1969). This accretion process, which is driven purely by the gravitational pull of the compact nuclear object, is known to be the most efficient way to convert a mass reservoir into heat, and then radiation. It is significantly more efficient than nuclear fusion in stars (Shapiro \&
Teukolski 1987). In the present-day Universe we have evidence that massive black holes also exit in galaxies with no signs of nuclear activity. This evidence comes from the dynamical study of the motion of stars and gas in galactic nuclei in the region where the central black hole dominate the gravitational potential (the, so, called, sphere of influence ${ }^{1}$ of the black hole). For the Galacic Center we have the most exquisite determination of the central black hole mass from studying individual orbits of stars well within the gravitational influence radius of the black hole (e.g., Gillessen et al. 2017). The combination of dynamical analysis for the study of "dormant" black holes, and the study of the frequency of AGN, led to the conclusion that massive black holes are ubiquitous at the center of galaxies over a broad range of masses (Kormendy \& Ho 2013), from the most massive early-type galaxies at the centers of groups and clusters to the relatively low-mass galaxies the size of the Large Magellanic Cloud (Pardo et al. 2016).

1 The black hole sphere of influence is typically defined to be a sphere with a radius $r_{h}=G M_{B H} / \sigma^{2}$, where $\sigma$ is the central velocity dispersion of the host galaxies. For a typical black hole of $10^{8} M_{\odot}$ in a galaxy of $\sigma \sim 200 \mathrm{~km} / \mathrm{s}$, the sphere of influence has a radius of $\sim 11 \mathrm{pc}$. 
Many models have been proposed for the origin of massive black holes (see the recent reviews by Volonteri 2012; Haiman 2013; Johnson \& Haardt 2016, and Latif \& Ferrara 2016), where the different formation scenarios postulate different types of precursors (or "seeds") for massive black holes. After formation, seed black holes start experiencing phases of exponential growth, which generally wash away direct information on the initial mass. The initial mass of the seeds depends on the type of precursor and on the processes associated to the formation of the black hole. The most straightforward, and widely explored, scenario assumes that the precursors of black hole seeds are Population III stars, the first generation of stars, appearing in the first hundred million years of the history of the Universe (e.g., Abel et a. 2002; Bromm \& Larson 2004; Greif et al 2010; Hirano et al. 2014). Despite the large masses reached by PopIII stars $\left(100-1000 M_{\odot}\right)$, the seed black holes resulting by the collapse of such stars would be orders of magnitude below the mass of supermassive black holes (Madau \& Rees 2001). Because of the nature of the precursor and the relatively small mass of the initial black hole, this scenario for the origin of massive black holes is usually referred to as "PopIII" or "light seeds" scenario. Alternative theories have explored models in which seeds form from the collapse of very large gas clouds, with a significantly larger initial mass $\left(>10^{4} M_{\odot}\right)$. Depending on the type of evolution of the precursor gas cloud, the upper limit for the seed mass could range from $10^{6} M_{\odot}$ to $10^{9} M_{\odot}$ in some very extreme scenarios (e.g., Mazzucchelli et al. 2017). These models, commonly referred to as "direct collapse" or "heavy seed" models, have gained more popularity in the recent years, as more evidence is building up on the existence of extremely massive black holes at very high redshifts, and such heavy seeds provide a significant "head start" for the mass build-up of the black holes powering the most luminous quasars. Indeed, one important constraint on formation scenarios comes from the existence of very bright quasars, with bolometric luminosities in the range $10^{47}-10^{48} \mathrm{erg} / \mathrm{s}$, which are already present at redshifts as high az $z \sim 7.1$ (Fan et al. 2006; Mortlock e al. 2011; Bañados et al. 2018). Even assuming that these quasars emit near the Eddington limit ${ }^{2}$, the masses inferred for the black holes are in excess of $10^{9} M_{\odot}$, namely comparable to the most massive black holes in the present-day Universe. The first problem that arises then is how to explain that such large masses can be achieved so early on. At $z=7.1$ only 0.5 Gyr have elapsed since the Big Bang. A simple calculation shows that one needs a seed black hole of at least $10^{4} M_{\odot}$ in order to be able to grow to such high masses while not overshooting the Eddington limit (see the discussion in Section 1.2).

Finally, another set of scenarios, which can lead to seeds with masses in between the Pop III and the direct collapse models, are those that involve core collapse

2 The Eddington limit expresses the balance between radiation pressure force and the inward gravitational pull exerted by the black hole. The Eddington luminosity, reached at the limit, is given by $L_{E d d}=4 \pi G M_{B H} \mu m_{p} c / \sigma_{T}$, where $M_{B H}$ is the black hole mass, $\mu$ is the mean molecular weight of the gas, $m_{p}$ is the mass of the proton and $\sigma_{T}$ is the Thomson scattering cross-section. For pure ionized hydrogen, the Eddington luminosity is: $L_{E d d}=$ $1.28 \times 10^{3} 8\left(M / M_{\odot}\right) \mathrm{ergs} / \mathrm{s}$. of a dense star cluster (e.g., Portegies Zwart et al. 2004; Devecchi et al. 2012). Under this framework, the seed arises from the runaway mergers of stellar mass black holes and/or the formation of a central Very Massive Star (VMS) from stellar mergers. Seeds with masses up to $1000 M_{\odot}$ can be formed in this scenario (see the review by Latif \& Ferrara 2016).

In the reminder of this section we will spend some time recalling the modern view on galactic structure and discussing quantitatively the important arguments concerning the accretion timescale of massive black holes as well as the general features of the Pop III seed scenario and of direct collapse scenarios. They will be instrumental to understand the astrophysical motivations behind our new direct collapse scenario.

\subsection{A few remarks on galactic structure, mass transport and scaling relations}

Our modern view of galaxies is that they comprise stars (distributed in different morphologies), interstellar gas, a dark halo, which is dominant in mass, and, in most cases, a central massive black hole. Our own Milky Way conforms with this general description. While star formation is a distributed process, namely stars form throughout the galaxy disk, massive black holes seem to be able to assemble only in galactic nuclei. Galactic nuclei contain typically less than $0.1 \%$ of the galaxy stellar mass in present-day galaxies even in galaxies containing the densest and more concentrated stellar components, namely those hosting a nuclear star cluster (eg., Walcher et al. 2005; Georgiev et al. 2016). Nearby examples are the nuclear star clusters in the late-type spiral galaxies M33 and NGC 4244 (Seth et al. 2008). Instead SMBHs often comprise a few percent (up to almost $10 \%$ in some cases) of the galaxy stellar mass (Kormendy \& Ho 2013). Above the mass scale of M31 galaxies that have both a nuclear star cluster and a central SMBH do indeed show that the latter has a mass up to 1000 times larger than the former (Figure 7 of Georgiev et al. 2016). In order for central massive holes to capture a much higher baryonic mass relative to nuclear star clusters, one obvious source of their efficient growth must be the interstellar gas, as this contributes at least a few percent of the stellar mass budget. The latter however, is mostly found outside the nuclear region. This leads to the notion of gas inflow, whereby baryonic matter at large radii is transferred to the nuclear region as long as it is able to lose angular momentum and sink. If star formation is negligible, such gas could all add up to the mass of the black hole. From this point of view, star formation and $\mathrm{BH}$ seed formation and/or growth can be viewed as competing processes.

We note that significant effort in the community has been devoted to devise scenarios in which star formation is heavily suppressed, thus allowing a larger fraction of baryons to turn into the central black holes seeds at early times. At the same time, as gas in galactic disks is prevented to fall inwards by rotational support, generating inflows to the center requires to find mechanisms to generate angular momentum transport (important also in the context of black hole feeding, see e.g., Jogee 2006). This is a very generic problem in many areas where astrophysical disks are relevant, the prototypical example being accretion disks of stars and compact objects, black holes included (e.g., Shapiro \& Teukolski 1987). 
The body of theoretical knowledge developed in the context of star formation and accretion disk physics will become relevant also for our purpose.

Relative to the mass budget of other components, at high redshift the conversion efficiency of baryonic matter might have been even more skewed in the direction of central black hole assembly, at least in the galactic hosts of high-z quasars. Indeed, although often not emphasized, the mass inferred for the black holes powering high-z quasars (up to $10^{9}-10^{10} M_{\odot}$ ), could be comparable to that of their host galaxies at that time, assuming that these galaxies are in the mass range of what can be inferred by abundance matching (e.g. Behroozi et al. $2013)$ given the halo masses $\left(10^{12}-10^{13} M_{\odot}\right)$ derived by quasar clustering studies (see, e.g., the recent results at $z>3$ of Timlin et al. 2017 and He et al. 2017). Given those numbers, it follows that at least $1 \%$ of the standard baryon budget of such halos is somehow converted into a massive black hole. The standard baryon budget here corresponds to assuming that halos capture their share of baryons in proportion to the cosmic baryon fraction suggested by different cosmological experiments (e.g. Planck results, Ade et al. 2015). This translates into each halo having captured a baryonic mass of about $1 / 6$ of its dark matter mass. Now, for halos of this mass various diagnostics, from abundance matching to weak lensing and clustering analysis, suggest that, at least up to $z \sim 5$, the stellar mass of the galaxies embedded in them is also in the range $2-6 \%$ of the halo mass (Behroozi et al. 2013; Moster et al. 2017). If these results can be extrapolated to $z>5$, which still awaits verification, it would mean that at early epochs the black hole formation efficiency could be as a high as the efficiency of the galaxy formation process itself. This is quite striking since at low redshifts galaxies of similar halo and stellar masses, such as the Milky Way, have black holes weighting only $10^{-6}$ of their halo mass. This seem to suggest that the processes at the origin of the high-z quasars could be different from those responsible for the main population of massive black holes, and perhaps quite exceptional. This could also imply that the correlations between black hole masses and galaxy properties, such as stellar mass or stellar velocity dispersion, widely observed in low redshift galaxies, may not yet be established at $z>5$ for the hosts of the bright quasars. While evidence of the latter is just beginning to be gathered, due to the obvious observational challenges, it is already clear that there can be substantial deviations from the standard correlations already at $z \sim 4$ (e.g., Trakhtenbrot et al. 2015).

\subsection{The assembly timescale of high-z quasars}

We briefly recall here the arguments that support the need for a massive $\mathrm{BH}$ seed in order to explain the rapid emergence of the bright high-z quasars. The time $t\left(M_{B H}\right)$ required for a black hole of initial mass $m_{\text {seed }}$ to reach a mass $M(B H)$, assuming Eddington-limited and continous accretion, is given by:

$$
t\left(M_{B H}\right)=\frac{t_{E d d}}{f_{\text {edd }}} \frac{\epsilon_{r}}{(1-\eta)} \ln \left(\frac{M_{B H}}{m_{\text {seed }}}\right),
$$

where $t_{E d d} \equiv c \sigma_{T} /\left(4 \pi G \mu m_{p}\right)$ is the Eddinton time $(\sim 0.45$ Gyr for a pure hydrogen gas, with $\mu=1), f_{\text {edd }}$ expresses at which fraction of the Eddington limit the black hole is accreting, and $\epsilon_{r}$ and $\eta$ are, respectively, the radiative efficiency and accretion efficiency ${ }^{3}$. Assuming a radiatively-efficient accretion event, we assume $\epsilon_{r}=$ $\eta=0.1$, a typical average value for Shakura-Sunyaev thin disks (Shakura \& Sunyaev 1973). Adopting a more realistic molecular weight per electron for a plasma at zero metallicity with cosmic abundance of hydrogen (X $=0.75)$ and helium $(\mathrm{Y}=0.25), \mu=1 /(1-Y / 2)=1.14$, the Eddington time lowers to $t_{E d d} \sim 0.39$ Gyr. Note that the precise value of the metallicity is marginal in this calculation, as metallicity has only a minor effect on the value of the molecular weight (e.g., for a gas at solar metallicity, $\mu=1.18$ instead of the value $\mu=1.14$ assumed here). The mass of the supermassive black holes powering the most luminous high-z quasars is of the order of $M_{B H}=10^{9} M_{\odot}$, so that this is the value we assume for the target mass. Further assuming that the seed black hole is able to accrete continuously at the Eddington limit $\left(f_{\text {edd }}=1\right)$, the timescale necessary to reach the target mass is $t_{\left(M_{B H}\right)}=0.4$ Gyr for a seed of $10^{5} M_{\odot}$, while is $t_{\left(M_{B H}\right)} \sim 0.7$ Gyr for a black holes starting from $10^{2} M_{\odot}$, the average value for light seeds produced by Pop III stars. In this latter case the growth timescale is comparable to the age of the universe at $z=7$ (assuming the Planck cosmology of Ade et al. 2015). Hence, the rapid appearance of quasars within the first billion years of the Universe can be more easily explained if the progenitors of these luminous first quasars are massive seeds, and that is why the community has devoted progressively more interest in models for the formation of massive seeds, as further pointed out in the rest of this introductory section.

\subsection{PopIII seeds}

The first generation of stars, the primordial Population III stars, formed in the first few hundreds million years after the Big Bang. They are expected to have been massive as the gas from which they formed was metalfree, thus unable, via the efficient metal cooling, to cool to low temperatures and fragment to the smaller scales typical of "normal" stars (e.g., Abel et a. 2000; Bromm \& Larson 2004; Greif et al. 2010). Indeed, in metalfree gas the only elements available are hydrogen and helium, which have limited radiative transitions compared to higher atomic number elements.

Therefore, both Pop III remnants seeds and seeds formed in star clusters, with masses typically below $1000 M_{\odot}$, might be generally too light to grow to the most massive black holes observed at high-z in the available time. If the Eddington limit is superseded, then the accretion flow should be stopped and an outflow should arise instead, as radiation pressure wins. Yet, this is true only in the simple spherical isotropic accretion context, as we will discuss further in Section 7 . The environments where Pop III BH seeds may form at $z \sim 15-30$ have been extensively studied with numerical simulations, which agree on the fact that such seeds grow too slow, at Sub-Eddington rates, as the gas in their

3 The accretion efficiency $\eta$ directly depends on the spin of the black hole, and reaches the maximum value of $\eta \sim 0.42$ for maximally rotating Kerr black holes. The radiative efficiency $\epsilon_{r}$ depends both on the type of accretion and on the accretion efficiency: for radiatively efficient accretion events, one can assume $\epsilon_{r}=\eta$. 
host mini-halo has been efficiently photoionized and/or expelled by the massive stellar progenitor (eg., Johnson \& Broom 2007; Wise et al. 2008). This is because the primordial stars in the mass range $140-260 M_{\odot}$ explode as pair-instability supernovae (Heger et al. 2003), liberating enough energy to effectively empty the mini-halos of gas. On the other side, the lower-mass PopIII would still damp significant energy, having a significant impact on the ambient medium if they form in clusters as it is likely (Greif 2015). Only later, when star formation has led to metal enrichment, increasing the cooling rate, and/or the original mini-halo has grown significantly in mass to allow atomic cooling via Ly-alpha radiation, accretion can in principle restart. If that happens, and Super-Eddington accretion can take place, fast growth might still be possible on much less than a Gyr timescales (Lupi et al. 2016). Inayoshi, Haiman and Ostriker (2016) found Super-Eddington flow steady-state solutions provided that the density of the surrounding gas is above a threshold density in order to trap photons. In this case, a black hole can grow continuously at very high rates even if accretion occurs spherically. Still, no direct radiation hydrodynamical calculation on nuclear or sub-nuclear scales exists that shows that this growth mode can be sustained up to the extremely large $\mathrm{BH}$ masses needed to explain the bright high-z quasars (see, e.g., the review by Johnson \& Haardt 2015). Therefore, the existence of high-z quasars so far argues in favour of some form of direct collapse. We note, however, that a broader, and less extreme, population of black holes might have evolved from PopIII remnants, as further discussed in Section 7. Two diverse channels of black hole formation could also explain the dearth of lower-luminosity quasars seen by Treister et al. 2013.

\subsection{The different flavours of direct collapse}

As mentioned above, with the term "direct collapse" the community generally refers to all the different models that predict the formation of a "heavy" seed, with initial mass above the approximate value of $10^{4} M_{\odot}$. Ironically, multiple stages are involved in the direct collapse scenarios, where the word direct refers to the notion that there is no preceding phase of mass growth starting of a lighter initial black hole. In this review we will focus on a particular flavour of direct collapse which is different from the mainstream one. But before entering into the details of this new model in the next section, we will first describe here the more popular version of direct collapse, as it will then be easier to appreciate the differences that our new model introduces.

\subsubsection{Direct collapse in metal-free protogalaxies}

In the standard picture, the key condition for the multi-scale gas inflow necessary to generate a central collapse into a massive black hole seed, is that fragmentation by gravitational instabilities should be suppressed. The rationale behind requiring such condition is that fragmentation would result into dense clumps that would produce regular stars, thus using up the gas that would have otherwise ended up in the protogalactic nucleus. In this picture, the formation of the black hole seed has to precede star formation. The necessity to avoid gas fragmentation translates into a condition on the cooling time of the gas. Indeed, it is a general result of gravitational instability theory in differentially rotating gas disks that fragmentation can only occur if local mass surface density is high enough, so that gravity wins over pressure and centrifugal forces, and if the local cooling time in such high surface density regions is shorter than the local orbital time (Gammie 2001; Lodato \& Natarajan 2006). While for some time it has been difficult to quantify exactly how strict such condition is in realistic three-dimensional disks, recent advances in numerical techniques have shown that the condition is indeed really strict once numerical sources of angular momentum loss are minimized (Deng, Mayer \& Meru 2017). In primordial gas conditions, radiative cooling is provided mostly by molecular hydrogen $\left(H_{2}\right)$ as the metallicity of the gas should be negligible, and thus the efficient cooling by fine structure lines of elements such as carbon and oxygen, so important in the local interstellar medium at the present time, is not relevant. This is why a major line of investigation has been, and still is, to devise ways by which molecular cooling can be stifled in protogalaxies. If this happens, it has been shown by multiple studies that collapse can take place nearly isothermally, at a temperature of the order of $10^{4} \mathrm{~K}$ (Regan et al. 2008; Choi et al. 2013;2015; Latif e al. 2013; Latif et al. 2015). The complete dissociation of molecular hydrogen requires the Lyman-Werner (LW) UV flux, namely photons in the $11.2-13.6 \mathrm{eV}$ energy band, to be above a certain threshold value, $J_{21}$, which depends on the radiation spectrum (it is lower for hotter radiation emitted by hotter, more massive stars) (Dijkstra et al. 2006; 2014; Agarwal et al. 2012; Latif et al. 2014; Visbal et al. 2014). Over the years, many studies aimed at quantifying the threshold flux have clarified that the cosmic ionizing background at $z>10$ is too low to meet the requirements. $\mathrm{H}_{2}$ dissociation could thus be possible only in the presence of localized powerful ionizing sources. An option could be and internal ionizing source, but that requires on-going star formation, which is what one wants to avoid in the first place in order to keep the gas metal-free. The more promising alternative are external localized sources, such as nearby star forming protogalaxy companions. The proximity and duration of exposure of the target galaxy to the luminous emitting nearby source (10-100 Myr) are crucial to achieve the desired dissociation, although recent works estimate that galaxy pairs with the required configuration should happen at least as frequently as the (rare) high-z quasars (Regan et al. 2017, but see Habouzit et al. 2016 for a possible tension between required number densities and probability of the required strength of the LW illuminating flux). Nevertheless, in none of the existing simulations, star formation in the galaxy source has been modelled in a fully self-consistent way nor the role of gas metallicity has been properly addressed. Latif et al. (2016) have shown that even with metallicities as small as $10^{-4}$ solar, inflow rates diminish drastically due to fragmentation and star formation if dust cooling is included. It is also still an open question whether or not, with a nearby star forming galaxy, the target galaxy can remain completely metal-free. Supernovae-driven outflows, which will frequently result as many of the massive stars producing the LW flux die out, can pollute the inter- 
galactic medium well beyond the host halo virial radius, and indeed this is considered the main driver of metal pollution of the Intergalactic Medium (e.g., Dave et al. 2001). Furthermore, the complete absence of fragmentation, even in metal-free, dissociated media, could not be a realistic outcome, as recent simulations show that the nuclei of protogalaxies are essentially rapidly rotating disks that, provided that the numerical model has enough resolution, can fragment into binaries or multiple objects at pc scales and below (Latif et al. 2015b). Interestingly, it has been argued that such clumps might merge quickly at the center of the system before regular star formation ensues, so that ultimately the same accumulation of gas can occur as when fragmentation is not present (Inayoshi \& Haiman 2014; Mayer et al. 2015).

Another potential issue with this conventional direct collapse picture is related to the rate of gas inflow. The estimated inflow rates are of the order of $0.1 M_{\odot} / y r$, peaking at $1 M_{\odot} /$ yr in some cases (e.g., Latif et al. 2015). While it is unclear how such rates vary on long timescales due to the limited time-baseline of the simulations, they are on the low-side compared to those required by models in which black hole seeds form from the collapse of protostars that avoid nuclear fusion, hence do not become supermassive stars, but rather quasi-stars (Begelman et al. 2006; Volonteri \& Begelman 2010), which allow fast growth of the initial black hole seed. Quasistars are radiation-pressure supported envelopes in quasiequilibrium with a central seed BH, essentially akin to giant stars in which the force supporting them against collapse is provided by accretion rather than fusion (Begelman et al. 2006; Begelman 2010). We defer the reader to section 5 for a detailed discussion of the various pathways to $\mathrm{BH}$ seed formation in direct collapse scenarios after a massive precursor has been assembled. Here we recall that very high inflow rates are favoured in certain pathways to generate massive $\mathrm{BHs}$, such as those in which the radial General Relativistic instabilities directly bring a supermassive cloud or protostar to dynamically collapse into a $\mathrm{BH}$ of the order of its mass, namely without prior formation of a supermassive star (Fowler \& Hoyle 1964; Shibata \& Shapiro 2002; Ferrara et al. 2014).

The reason why the estimated inflow rates in the traditional direct collapse scenario are relatively low, is due to the fact that this model of direct collapse has to take place at high-z $(z>15-20)$, so that the requirement of primordial gas metallicity can be satisfied. At such redshifts, the possible host for direct collapse would be the atomic-cooling halos, in the range $10^{8}-10^{9} M_{\odot}$. As explained in the next section, simple scaling arguments can also be used to recover the relatively-low inflow rates found in the simulations for halos in this mass range.

\subsubsection{The new direct collapse model}

A few years ago we began considering a whole different scenario for the emergence of direct collapse seeds, in which no particular restrictions on the thermodynamical conditions of the gas phase are required. Instead, the model relies on a particular trigger of multi-scale inflows: galaxy mergers (Mayer et al. 2010). In this alternative picture, which is the focus of this article, the event generating the conditions for $\mathrm{BH}$ seed formation in the galactic nucleus is the major merger between two massive gas-rich galaxies. Star forming galaxies with stellar masses comparable to the ones of massive spirals, such as our own Milky Way at the present day, already exist at $z \sim 10$. Their host halo masses are correspondingly 3-4 orders of magnitude higher than the halo masses typically assumed in simulations of metal-free protogalaxies, being in the range $10^{11}-10^{12} M_{\odot}$ as opposed to $10^{8}-10^{9} M_{\odot}$. These massive galaxies evolve in highly biased regions where the metal pollution by previous generation of stars has proceeded already, hence the interstellar medium has a composition akin to normal present-day galaxies, which implies efficient radiative cooling via metal lines (Mayer et al 2015). This is a major difference relative to the standard direct collapse route. Measurements of the gas metallicity in the hosts of some of the bright high-z quasars, via detection of the CO molecule, do exist (Walter et al. 2004). They imply, indeed, gas with already solar metallicity. Note that highly biased regions, corresponding to density fluctuations whose amplitude is significantly higher than the mean amplitude (4-5 $\sigma$ peaks in technical jargon ${ }^{4}$ ) are also implying the high clustering amplitude of the high-z quasars. Cosmological simulations that assume pre-existing massive $\mathrm{BH}$ seeds in the range $10^{4}-10^{5} M_{\odot}$ and concentrate on modelling their growth can do it successfully in such highly biased regions as accretion of cold gas funnelled by cosmological filaments is very high (e.g., Di Matteo et al. 2012; Feng et al. 2014; Di Matteo et al. 2017). Furthermore, major mergers do occur at $z \sim 8-12$ in these simulations as such massive early galaxies assemble (Di Matteo et al. 2012), precisely because the galaxy formation process is accelerated in high sigma peaks (Wilkins et al. 2017). In essence, our new scenario for the efficient accumulation of gas in the center of high-z massive galaxies, connects naturally with the models of fast growth of massive black holes, which successfully reproduce the rapid appearance of bright quasars by $z \sim 7$, exploiting the nature of the special environments in which quasars appear to exist at high redshift. We finally underline that the high-mass halos involved in this model are also able to sustain gas inflow rates that are significantly higher than the ones possible in $z \sim 15-20$ protogalaxies. This, as we will further see in the next section, has paramount consequences on the nature of gas collapse at small scales, and thus on the nature of the precursor of the $\mathrm{BH}$ seed.

The rest of the article is structures as follows. In the next two sections we review the details of our mergerdriven model for direct collapse, providing also a deeper look at the physics involved than in the specialized publications already published. We start by elucidating important notions, such as the connection between gas infall rates and host halo potential well and, most importantly, how the emergence of a dense, optically thick nuclear gas disk stable to fragmentation might be the necessary consequence of heavy loading via high inflow rates. This will be the rationale behind our novel analytical model

\footnotetext{
${ }^{4}$ In a hierarchical structure formation scenario, structures forms from small density fluctuations that grow as the Universe evolves. The amplitude of the fluctuations is defined as $\nu \equiv \delta_{c} / \sigma(M, z)$, where $\delta_{c} \sim 1.69$ and $\sigma(M, z)$ is the rms variance of the linear density field smoothed on a scale $R(M)=\left(3 M / 4 \pi \rho_{m}\right)^{(1 / 3)}$ (Press \& Schechter 1974).
} 
of massive loaded nuclear disks presented in section 2 . With these premises it will be easier to understand the numerical results of our 3D hydrodynamical simulations reported in section 3 . In section 4 we will outline the possible pathways for the evolution of central massive central baryonic objects towards a massive $\mathrm{BH}$ seed. We will then connect our results to the large-scale cosmological context in section 5, where we present a semianalytical model of galaxy formation equipped with our direct collapse scenario to study how well we can reproduce not only the high-z quasars but also the many other observables associated with the population of luminous AGNs as well as non-accreting massive BHs at both high and low redshift. Finally, section 6 will contain a discussion of alternative rapid $\mathrm{BH}$ growth models, focusing on Super-Eddington accretion, and will summarize the observational challenges in constraining the various scenarios, and ours in particular.

\section{THE PHYSICS OF MULTI-SCALE GAS INFLOWS IN HIGH-REDSHIFT GALAXIES}

In this section we review the physical processes related to the formation of protogalactic disks during the first phases of structure formation, and we will summarize the basic concepts related to the stability and evolution of these disks in the case of no external perturbations. In Section 3 and 4 we will see how this picture changes for the case of a nuclear disk formed during a major galaxy merger, in which the infall of gas from galactic scales has a substantial effect on its dynamical evolution. Section 4 , in particular, will present a simple analytical framework that attempts to address the physics behind the simulations described in section 3 , giving rise to what we will dub "the loaded disk model".

\subsection{Inflows at supra-galactic scales: from the dark halo to the protogalactic disk}

The first step that needs to be accomplished by any direct collapse scenario is to efficiently bring interstellar gas from kiloparsec scales to parsecs scales and below. This is a necessary stage to achieve the formation of a massive, ultra-dense baryonic core, a potential precursor of the BH seed. The framework within which this has to be studied is the modern theory of galaxy formation within the Cold Dark Matter (CDM) cosmological model (White \& Rees 1978). In CDM, cosmic structures form hierararchically, in a bottom-up fashion, from gravitational instability of small initial density perturbations in a dissipationless dark matter component, which eventually grow into galaxy-sized dark halos by via accretion of matter and mergers with other halos (Blumenthal et al. 1980; White \& Frenk 1991). In CDM the predicted size scale of dark halos, the virial radius $R_{v i r}$, embedding typical present-day galaxies such as our own Milky Way is in the range 200-300 kpc, and such halos have characteristic virial masses $M_{v i r} \sim 10^{11}-10^{12} M_{\odot}$ (precisely, these are the typical numbers that apply for a CDM model with a dominant contribution of the cosmological constant at low redshift, as it is in the case of our Universe). At higher redshift, since the mean density of the Universe is higher, halos collapse at a higher characteristic density, resulting in a smaller $R_{v i r}$, which roughly scales as $1 /(1+z)^{3 / 2}$. Baryonic matter falls into DM halos due to their gravitational attraction, cooling and forming a central rotating disk due to conservation of an initial small amount of angular momentum imparted by tidal torques (White \& Rees 1978).

The simplest approach to evaluate the importance of gas inflows in galaxy-scale systems is to assume that gas is freely-falling inside the potential well of the host dark matter halo. In this case, one can borrow the basic concepts of the so called inside-out collapse framework of star formation theory (eg., Stahler \& Palla 2010). We thus envision that baryons first accrete in free-fall to the center of the halo from the cosmic web, and, after a sizable mass has gathered, sustained accretion onto the baryonic core continues due to its own gravitational pull. Inside-out collapse refers to this second phase. This simple model is reasonable in systems where (i) the angular momentum is negligible and (ii) the cooling is efficient enough to yield nearly isothermal conditions even in the presence of shocks or other heating mechanisms during infall. About the first requirement, spins of halos in CDM are small at the virial radius scale, but eventually angular momentum has a major impact on the gas flow at smaller scales, where a disk forms as fluid elements reach the radius of centrifugal support (White \& Rees 1978). This essentially means that the model will break down inside the protogalactic disk. Note also that in CDM the spin of baryons is essentially imposed by the spin of the underlying dark matter distribution, and has a well defined universal distribution (eg Bullock et al. 2001a) yielding a centrifugal radius that is always $4-5 \%$ of the virial radius $R_{v i r}$. This implies that, for galactic halos, the centrifugally supported disk that forms should have a scale of $10-15 \mathrm{kpc}$, roughly consistent with present-day observed galaxy sizes, but would be much more compact in protogalaxies at high-z, $1-1.5 \mathrm{kpc}$ at $\mathrm{z}=8$ for example, because of the reduction of $R_{v i r}$ (Mo, Mao \& White. 1998). The second condition, requiring the gas to be isothermal during free-fall, can be satisfied in cosmological filamentary accretion from the cosmic web, the so called cold mode accretion, which is believed to be the main mode of galaxy formation at least up to halo virial masses of nearly $10^{12} M_{\odot}$ (see Keres et al. 2009). Cold flow accretion is indeed the result of radiative cooling overtaking shock heating to zero-th order (White \& Frenk 1991; Dekel \& Birnboim 2006) so that a stable hot gaseous halo cannot form below the aforementioned mass threshold. At very low mass scales photoionization by the cosmic ultraviolet background would stop or at least slow down the infall, but this is relevant after reionization, namely below the $z=8-20$ redshift range that $\mathrm{BH}$ seed formation mechanisms usually consider.

At the boundary of the protogalactic disks we can determine the gas infall rate in the following way:

$$
\dot{M}=\alpha V_{f f}^{3} / G
$$

where the free-fall velocity is $V_{f f} \sim V_{\text {circ }}$, with $V_{\text {circ }}$ being the so-called halo circular velocity, namely the velocity that a gas parcel would have on a circular orbit at a specific radius in the halo potential well (neglecting corrections due to the additional mass of the baryons), i.e. $V_{\text {circ }}(R)=\sqrt{(G M(R) / R)}$, where $G$ is the gravitational 
constant, and $M(R)$ the enclosed mass of the system at the radius $R$. Finally, $\alpha$ parametrizes the viscosity of the flow (see Lodato \& Natarajan 2006). We will also assume that $V_{\text {circ }} \sim V_{\text {vir }}$, where $V_{v i r}$ is the virial velocity of the dark halo, namely the characteristic velocity scale resulting by the gravitational collapse of collisionless dark matter in an overdensity with a characteristic virial mass $M_{v i r}$. This approximation neglects that the circular velocity is not a constant, but first raises, and then decreases at large radii in CDM halos reaching $V_{v i r}$ at the halo boundary (Mo, Mao \& White 1998). Yet, such variations do not exceed a factor of 2-3 at the scales of interest, hence can be safely neglected. As the overdensity amplitude sufficient for collapse depends on cosmological density parameters, the above scaling equations will depend on cosmology in detail. When we provide specific numbers in this review we will always assume the concordance cosmological model with matter density parameter $\Omega_{M}=0.3$ and cosmological constant density parameter $\Omega_{\Lambda}=0.7$ Cosmological scaling laws between dark halo global parameters, such as virial mass, virial radius and circular velocity, imply that $V_{v i r} \sim M_{v i r}^{1 / 3}$ (Mo, Mao \& White 1998). Moreover, we can assume $\alpha=1$ for the infalling matter described by eq.(1), as there is no natural source of bulk viscosity that would slow down the flow. Instead, at smaller scales, if a gravoturbulent protogalactic disk is established, a more appropriate value for the viscosity parameter is $\alpha=0.1$ (see section 4 for a detailed justification of how to choose the viscosity parameter).

Thus, assuming $c_{s} \sim V_{v i r} \sim M_{v i r}^{1 / 3}$ in eq. 3, yields that $\dot{M} \propto M_{v i r}$, highlighting that the inflow rate depends on the mass of the halo linearly. This has important implications as it suggests that much larger inflow rate can be sustained in higher mass halos. As in CDM theory cosmic structures form hierarchically, in a bottomup fashion, more massive halos form as time progresses, suggesting that larger inflow rates are achieved at lower redshifts. At $z \sim 15-20$, the typical redshift range considered in studies of first objects, the largest halos that can cool via atomic cooling, will have masses between $10^{9}$ and $10^{10} M_{\odot}$, while at $z \sim 8$ the first halos with virial masses of order $10^{12} M_{\odot}$ already appear, corresponding to $4-5 \sigma$ density fluctuations above the typical amplitude (Di Matteo et al. 2012, Feng et al. 2016). An increase of 2-3 orders of magnitude in halo masses means thus a corresponding increase of 2-3 orders of magnitude in inflow rate. Simulations of protogalaxies at $z \sim 15-20$ show that inflow rates are of the order of $1 M_{\odot} / \mathrm{yr}$ in $10^{7} M_{\odot}-10^{8} M_{\odot}$ halos, hence at $z \sim 8$, if our scaling arguments apply, inflow rates could in principle increase to $>1000 M_{\odot} /$ yr in $10^{12} M_{\odot}$ halos. Once the gas joins the proto-disk, viscosity could reduce the inflow rate by an order of magnitude (see also section 4), but in absolute terms the gas supply rate would still be remarkable. This shows the potential advantage of considering lower redshift, more massive halos in triggering prominent inflows. Such prominent inflows could in principle assemble a sizable central cloud by accumulating matter in the nucleus of the protogalaxy, which could then produce a massive $\mathrm{BH}$ seed in various ways (see section 5). However, since CDM halos have the same statistical distribution of spins irrespective of virial mass, the radius of centrifugal support is typically $3-4 \%$ of the virial radius $R_{v i r}$ at all mass scales, with $R_{v i r} \sim V_{v i r}$ (Mo et al. 1998). As a result, in higher mass halos the centrifugal barrier occurs at a much larger scales, which could potentially prevent the inflow from reaching pc scales and below. This is true unless some mechanism causes a catastrophic loss of angular momentum of the baryons, essentially reducing the centrifugal radius by orders of magnitude. As the simulations in section 3 demonstrate, this is what the strong torques and hydrodynamical shocks in the merger between two nearly equal mass galaxies can do (Kazantzidis et al. 2005), The conventional argument, though, in discarding lower redshift halos has always been that these would be metal-enriched, undergo then fast cooling and fragmentation, which would stifle inflows. Before going into the specific case of our merger model we will discuss, in the next section, how this argument on fragmentation is misleading since it neglects the rich and complex dynamics of self-gravitating flows. Self-gravitating disks have been widely studied with 3D simulations in the case of star and planet formation (Durisen et al. 2007). We can borrow many useful results and knowledge from these other fields.

\subsection{Stability and fragmentation of self-gravitating disks}

As we explained in the Section 1.4, the efficiency of radiative cooling plays a key role in conventional direct collapse models because it is directly tied to the susceptibility to fragmentation. How much a rotating, selfgravitating fluid system is prone to fragmentation, can be described by the combination of i) the Toomre Q parameter (Toomre 1964) parameter, which describes the balance between the stabilizing and de-stabilizing forces acting on the fluid and ii) considerations on the ability of the system to remain in a stable or unstable state based on its radiative cooling rate (Gammie 2001). The Toomre Q parameter is defined as $Q=\kappa c_{s} / \pi G \Sigma$, and can be derived using the framework of linear perturbation theory assuming a local axisymmetric perturbation in a razor-thin rotating axisymmetric disk (essentially a two-dimensional self-gravitating fluid, see Binney \& Tremaine 1987). Here $\kappa$ is the epicyclic frequency, which is of the order of the angular frequency of the fluid elements (so expresses how fast they move due to rotation), $c_{s}$ is the thermal sound speed, hence encapsulates the effect of thermal pressure, ad $\Sigma$ is the disk surface density, accounting for the self-gravity of the system ( $G$ is the gravitational constant). The Toomre stability analysis shows how, under a small initial perturbation, short wavelengths are stabilized by thermal pressure and long wavelengths are stabilized by rotation. The system is probe to collapse if $Q<1$, while it is stable if $Q>1$. The conditions in the definition of $Q$ are all local in an axisymmetric system, which implies that the parameter can be used in any system irrespective of its global mass and internal velocity distribution. While this is useful because it means the definition has general validity, it also means that when one uses $Q$ in a global disk model, a conventional procedure in the astrophysical literature, the Toomre parameter should be viewed as a phenomenological parameter which is not rigorous anymore and whose interpretation requires caution (often this is aided a posteriori by numerical simulations). Once $Q<1$, whether local collapse occurs or not depends on the sec- 
ond condition, the so-called Gammie criterion, whereby the radiative cooling timescale has to be comparable or shorter than the local orbital time. Local collapse, often referred to as fragmentation, can indeed occur only if the heat gained while the gas tries to compress in order to collapse gravitationally is removed rapidly enough by radiative cooling. The local orbital time measures the speed at which gas is gravitationally pulled together in the attempt to collapse (formally it is the characteristic dynamical timescale of the disk annulus with radius $R$ which $Q<1$ ). If not collapse is halted and the disk becomes stable as a result of heating $(Q>1)$. Note that the heat here comes from the action of self-gravity itself which triggers the compression, not by an external agent (this is not $t$ necessarily true in a disk that is externally perturbed).

There are important nonlinear aspects of selfgravitating disks that the Toomre analysis cannot capture, some related also to the global flow properties that cannot be explained with a local approach. For example, when $Q$ approaches 1 from above in a disk annulus, shocks occur at the interface between the overdense regions that are becoming unstable, such as spiral arms, and the background flow. In such a system fluid elements do not move on circular orbits as assumed for the unperturbed fluid background in the Toomre analysis. As a result, the gas velocity field tends to become chaotic from orderly even before $Q$ drops below unity, with its random kinetic energy growing at the expense of its initial gravitational potential and rotational energy. This state is referred to as gravitoturbulent. Efficient angular momentum transport is possible in this case (eg Binney \& Tremaine 2003), as in any turbulent fluid. However, here, at variance with conventional hydrodynamical turbulence, the energy source comes from self-gravity and the injection scale of turbulence is associated to the wavelengths of the perturbations that can become unstable based on the Toomre analysis. Largest wavelength modes, such as global spiral patterns, have wavelengths as large as a fraction of the disk radius. This means the injection scale can be quite large, which is why angular momentum transport is self-gravitating disks has a global character. As long as dissipation is sufficiently local, gravitoturbulence itself can be described as a source of effective viscous heating which leads to self-regulation of disks towards marginal stability ( Lin \& Pringle 1987; Lodato \& Rice 2004;2005). Other departures from the Toomre analysis are due to the fact that disks have a finite thickness. Numerical simulations that can follow non-linear stages of the instability and can do that in a global, three-dimensional set-up, find that $Q<0.7$ rather than $Q<1$ is necessary for fragmentation, namely a more restrictive condition that for the razor-thin disks assumed in the Toomre analysis (Nelson et al. 1998; Mayer et al 2004; Durisen et al. 2007). At least in keplerian disks simulations also find the system is driven quickly to fragmentation a global sustained spiral pattern develops where $Q<1.4$ somewhere (Mayer et al. 2004; Durisen et al. 2007; Kratter \& Lodato 2016).

Recently substantial work has been devoted to pin down the critical cooling timescale for fragmentation. In recent past different works in the context of protoplanetary disks have shown non-convergence of results with increasing numerical resolution, which has cast doubts on the physical understanding of the fragmentation process. The tendency has been that the critical cooling time for fragmentation was increasing by an order of magnitude as resolution was increasing by two orders of magnitude (e.g., Meru \& Bate 2011; 2012). However, recently evidence has been gathered that non-convergence is likely caused by numerical inaccuracies in capturing the strongly non-linear regime, with potential difference sources of errors coming from different aspects of numerical schemes. One prime example is numerical viscosity, which is an explicit term in the fluid equations in particlebased codes such as as Smoothed Particle Hydrodynamics $(\mathrm{SPH})$ but also in many popular finite volume codes designed to model disks (Meru \& Bate 2012). Very recent work employing more modern numerical techniques, such as the lagrangian mesh-less finite mass method (MFM) in the GIZMO code (Hopkins et al. 2015), that can follow more accurately the evolution of flow vorticity, and thus angular momentum transport, have finally recovered convergence and determined that the critical cooling time for fragmentation is short, being essentially equal to the local orbital time (Deng, Mayer \& Meru 2017). This agrees with the original results of Gammie (2001) obtained with $2 \mathrm{D}$ shearing sheets. It implies fragmentation is harder to attain than previously thought.

In summary, based on the discussion above threedimensional isolated disks, including protogalactic disks, could sustain efficient angular momentum transport without fragmenting down to really low values of the Toomre parameter, $Q<0.7$. Since protoagalactic disks are not keplerian, whether or not $Q<1.4$ marks the likely fragmentation threshold in this case is unclear. Also, as time progresses and protoagalactic disks become more metal-rich the radiative cooling timescale could shorten if gas is optically thin, which should favour fragmentation. In present-day galaxies, indeed the dominant neutral gas phase has a low-density and is optically thin, and indeed Giant Molecular Clouds (GMCs) are believed to form from gas disk fragmentation (e.g., Agertz et al. 2009). On the other end, if at high redshift disks are more gas-rich and denser, and thus become optically thick, this would lengthen the cooling timescale, which would have the opposite effect (in this case an increasing metallicity would favour stability even further as it would increase gas opacities, which in an optically thick system results in longer cooling time). The properties of the interstellar medium are thus crucial in this context. We will revisit this aspect in the next two sections, in which we will also drop the assumption of an isolated, unperturbed disk.

\section{SIMULATIONS OF THE MERGER-DRIVEN DIRECT COLLAPSE SCENARIO}

The merger driven model has been investigated with simulations of binary mergers between massive gas-rich disks whose structure is similar to present-day spirals (Kazantzidis et al. 2005; Mayer et al. 2010,2015, hereafter MA10 and MA15, respectively). For the simulations we employed the SPH codes GASOLINE and GASOLINE2 (Wadsley et al. 2004; Keller et al. 2014). The choice of modeling galaxies based on prexent-day disks is a simplification as we have observational evidence that, already at $z \sim 2-3$, massive gas-rich galaxies appear much more irregular in structure and turbulent relative A turbulent disk could boost certain processes occur- 

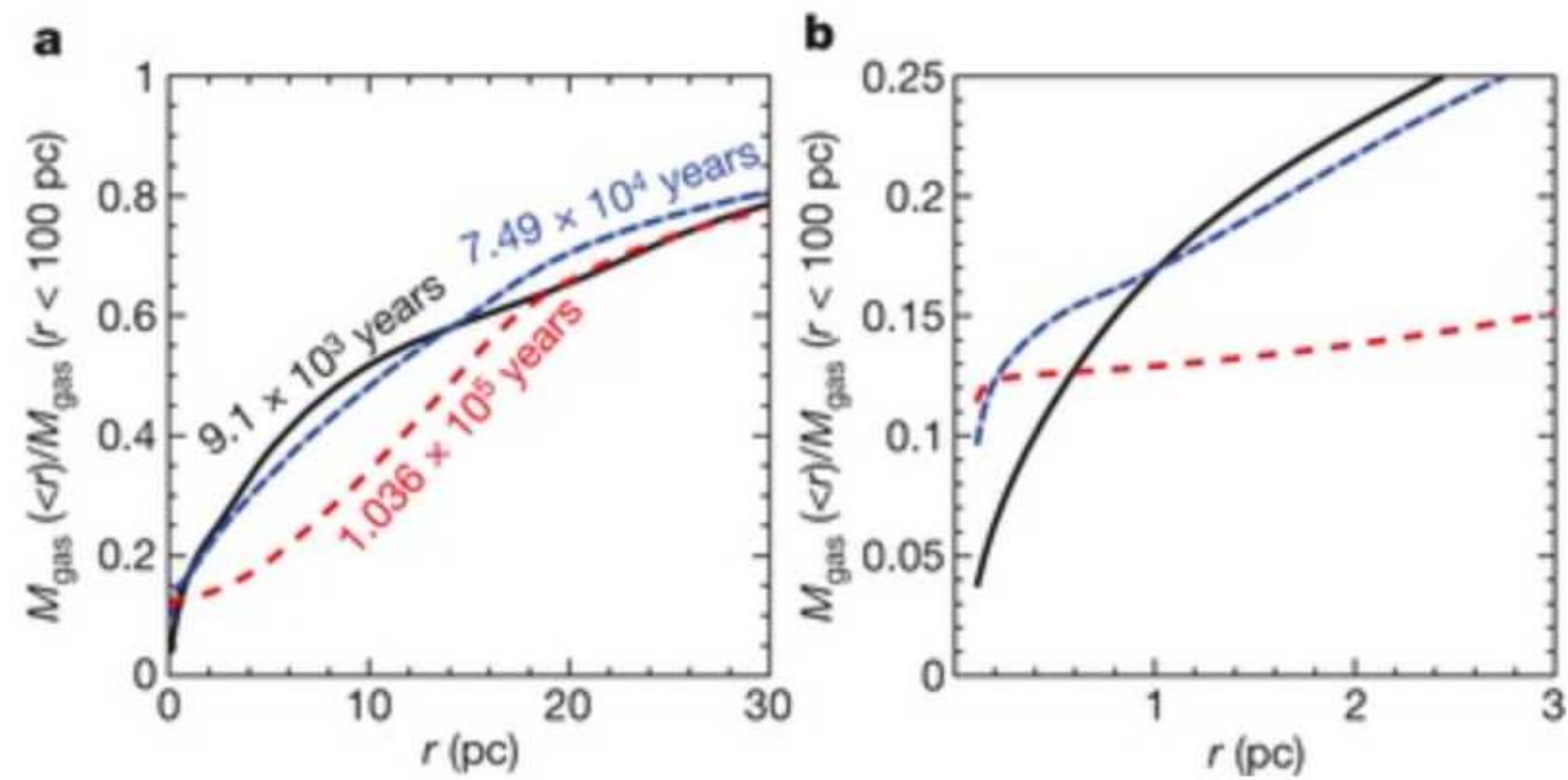

Figure 1. The figure shows the growth of the central gas mass distribution following the multi-scale galaxy merger in the MA10 paper, at tens of parsecs (left) and at parsec scales (right). Different curves correspond to different times starting from the completion of the galaxy merger as indicated in the labels. Adapted from MA10.

ring in gas flows that are crucial to achieve the prominent multi-scale inflows of our scenario. We will discuss these aspects later on in section 7 along with the prospects of incorporating them by starting directly from cosmological simulations of massive galaxies. New realistic cosmological hydro simulations of $z \sim 10$ mergers in highly biased regions are indeed in progress (Capelo, Mayer et al., in preparation). Among the simulations published so far the earliest ones adopted simplified thermodynamics, employing a fixed effective equation of state, while more recent ones included radiative cooling and heating explicitly. In the next two subsections we describe both types of simulations.

All our published simulations adopted the same initial conditions for the model galaxies. The two galaxy models employed in the simulations of major galaxy mergers represent identical multi-component systems with virial masses of $M_{v i r}=10^{12} M \odot$. They are constructed following the method by Hernquist (1993) and choosing the structural parameters in agreement with the scaling laws predicted by the $\Lambda$ CDM model (Mo et al. 1998; Kazantzidis et al. 2005). The baryonic disk is embedded in a dark matter halo that follows the NavarroFrenk-White (Navarro et al. 1996) profile with concentration $c=12$. The stellar and gaseous disk follow the same exponential density distribution, and a stellar bulge is also included. The stellar disk has a mass of $M_{\text {disk }}=6 \times 10^{10} M_{\odot}$, a scale radius of $R_{\text {disk }}=3.5$ $\mathrm{kpc}$, and a scale height of $z_{\text {disk }}=350 \mathrm{pc}$. The gaseous disk has the same scale lengths as the stellar disk. The gas mass, which is gradually reduced by star formation, is still about $10 \%$ of the disk mass at the time of the last pericenter passage. The bulge is a Hernquist (1990) model with $1 / 5$ of the disk mass and a scale length of 0.7 kpc. We refer to MA10 for more details on the numerical parameters of the large-scale merger simulation (see also Kazantzidis et al. 2005; Mayer et al. 2007). We note that the adopted virial mass is comparable to that of a Milky-
Way-sized galaxy at $\mathrm{z}=0$, and it corresponds to $\sim 5 \sigma$ dark matter fluctuations at $z=8-9$ in the WMAP9 cosmology (the cosmology assumed in the simulations). With the assigned disk and bulge mass the galaxy is consistent with the stellar-to-halo mass relation constrained by abundance matching and other statistical measurements correlating galaxy and halo properties up to very high redshift (Behroozi et al. 2013; Moster et al. 2017), although we caution that no robust measurements exist at $z>7$. The galaxies are placed on a parabolic orbit whose parameters are chosen to be typical of halo/galaxy pairs in large cosmological volumes (Kochfar \& Burkert 2006).

In order to follow the gas inflow to sub-pc scales we had to increase the resolution of the hydrodynamical merger simulations in subsequent steps. We split individual gas particles within a spherical volume of radius $30 \mathrm{kpc}$ into eight child particles in two subsequent steps. We correspondingly reduce their gravitational softening, achieving a final resolution of $\sim 2600 M_{\odot}$ and $0.1 \mathrm{pc}$. Pre-existing star particles and dark matter particles are not split, and their softening is left unchanged. They essentially provide a smooth background potential to avoid spurious two-body heating against the much lighter gas particles. The momentum conserving splitting procedure, is described in Roskar et al. (2015). Note that in the earlier, non-radiative simulations of MA10 a simpler splitting algorithm exploiting only a gather SPH kernel operation was employed . Later we have found out that using a scatter operation is numerically more robust when re-assigning internal energies to child particles in presence of steep temperature gradients (in MA10 this was not important as the use of an effective equation of state was leading by construction to weak local temperature gradients). We point the reader to Roskar et al (2015) for a detailed description of the updated particle splitting algorithm. The choices of when to split the gas particles and the size of the splitting volume are the same 
in both MA10 and MA15 and are discussed in the Supplementary Information of MA10. They are motivated by inducing minimal numerical fluctuations. To this end the refined region was large enough to avoid any contamination of low-res particles for the entire duration of the simulations.

After the two galaxy cores reached a separation $<100$ $\mathrm{pc}$, most of the gaseous mass is collected in the inner $100 \mathrm{pc}$ volume and it is traced with more than a million gas particles. By performing numerical tests, we have verified that, owing to the fact that gas dominates the mass and dynamics of the nuclear region, the large softening adopted for the dark matter particles does not significantly affect the density profile of the inner dark halo that surrounds the nuclear disk. These and other numerical tests showing the robustness of the technique were presented in Mayer et al. (2007).

\subsection{Non-radiative simulations}

In MA10 we simulated multi-scale gas inflows in equal mass mergers between two identical galaxies modelled with the initial conditions described in the previous section. The SPH code GASOLINE was used to model the combined hydrodynamical and gravitational physics, while radiation was not treated explicitly, as we explain below. Star formation and thermal feedback from Supernovae Type II were included only in the large scale merger simulation but not in the re-sampled simulation stage after particle splitting was applied (see MA10 for details). We also performed two additional simulations of equal mass merging galaxies in which the mass of the two galaxies was reduced by a factor of 5 and 20, respectively. These addition models were constructed by simply scaling the radius and velocity in order to preserve the same density and virial equilibrium condition. This is consistent with the notion that, for a given cosmology and redshift, the critical overdensity for collapse of dissipationsless cold dark matter is fixed (Mo et al. 1998). This yields scaling relations between virial mass $M_{v i r}$, virial radius $R_{v i r}$ and virial velocity $V_{v i r}$ in the form $M_{v i r} \sim R_{v i r}^{1 / 3}$ and $V_{v i r} \sim R_{v i r}$. Central in the MA10 work was the two-step procedure in running the simulation to capture sub-pc scale dynamics while starting from a galaxy merger in which the initial dark matter halos extended out to hundreds of kpc. The other central aspect was the adoption of simplified thermodynamics of the gas. We employed an adiabatic equation of state with variable adiabatic index $\gamma$, of the form $P=(\gamma-1) \rho u$, where $P$ is the thermal pressure, $u$ is the internal energy (the gas is assumed to be ideal), and $\rho$ is the density. The range of $\gamma$ values adopted was determined based on the density range in the simulations, and was based on a simple equilibrium model of the ISM illuminated by a ionizing flux produced by local star formation (Spaans \& Silk 2000). The internal energy equation was solved, thus capturing irreversible heating from shocks, which is important in galaxy mergers.

In the first stage of the mergers dynamical friction between the two extended dark matter halos erodes the orbital energy of the two galaxies, which sink towards one another and merge in less than a Gyr. When the two galaxies are at a distance of about $5 \mathrm{kpc}$ we applied the particle splitting technique and decrease the gravi- tational softening in two subsequent steps (from 100 pc to 5 , and then to $0.1 \mathrm{pc}$ ). The final collision of the two galactic cores produces a massive turbulent rotating nuclear disk with a mass of about two billion solar masses and a radius of about $80 \mathrm{pc}$. The disk is born in an unstable configuration, with a prominent two-armed spiral pattern imprinted by the collision and sustained by its own strong self-gravity. The gas has a high turbulent velocity $(100 \mathrm{~km} / \mathrm{s})$, whose gravitourbulent origin we discuss in the next section, and rotates at a speed of several hundred kilometres per second within 50 pc. The disk is stable against fragmentation but the strong spiral pattern swiftly transports mass inward and angular momentum outward About 10,000 years after the merger is completed more than $20 \%$ of the disk mass has already accumulated within the central few parsecs (Figure 1) where the inflow rate peaks at over $10^{4} M_{\odot}$ per year, orders of magnitude above inflow rates in isolated protogalaxies (see sections 1-2).

The gas funnelled to the central region (2-3 parsecs) of the nuclear disk settles into a rotating, pressuresupported cloud. The density of the cloud continuously increases as it gains mass from the inflow until it becomes Jeans unstable and collapses to sub-parsec scales on the local dynamical time of 1000 years (see Figure 2). The supermassive cloud contains around $13 \%$ of the disk mass (Figure 1). The simulation is stopped once the central cloud has contracted to a size comparable to the spatial resolution limit. At this point the cloud is still Jeans unstable. With greater resolution its collapse should continue because the equation of state would become essentially isothermal at even higher densities. With its steep density profile, a power law of density with exponent steeper than -2 , at radii as small as a thousandth of a parsec the cloud would be as massive and dense as a protostellar envelope that can collapse directly into a massive black hole seed embedded in a gaseous envelope, the so-called quasi-star proposed by Begelman and collaborators (Begelman et al. 2006; Begelman \& Volonteri 2010). Other routes, such a supermassive star which later collapses into a massive black hole encompassing a large fraction of its mass, or even direct formation of a supermassive black hole via the relativistic radial instability, are also possible. We will discuss pathways more in detail in section 5 using the newer set of radiative simulations.

The formation of the collapsing cloud occurs in only $10^{5}$ years after the completion of the merger, a timescale much shorter than the $10^{8}$ years needed to convert most of the nuclear gas into stars during the starburst that accompanies the merger. From such earlier simulations it was already clear that with such short formation timescales for the massive $\mathrm{BH}$ precursor object one overcomes a major problem of the more conventional direct collapse scenarios, namely the need to suppress cooling and star formation. Simply gas inflows occur on a timescale shorter than the typical gas consumption timescale into stars. This point will be strengthened further with the radiative simulations described in the next section, in which we will see that, additionally, the very inner dense pc-scale region is completely stable to fragmentation and star formation even when all cooling channels are active. The high inflow rates measured suggest the simulated system belongs to the heavily mass loaded 


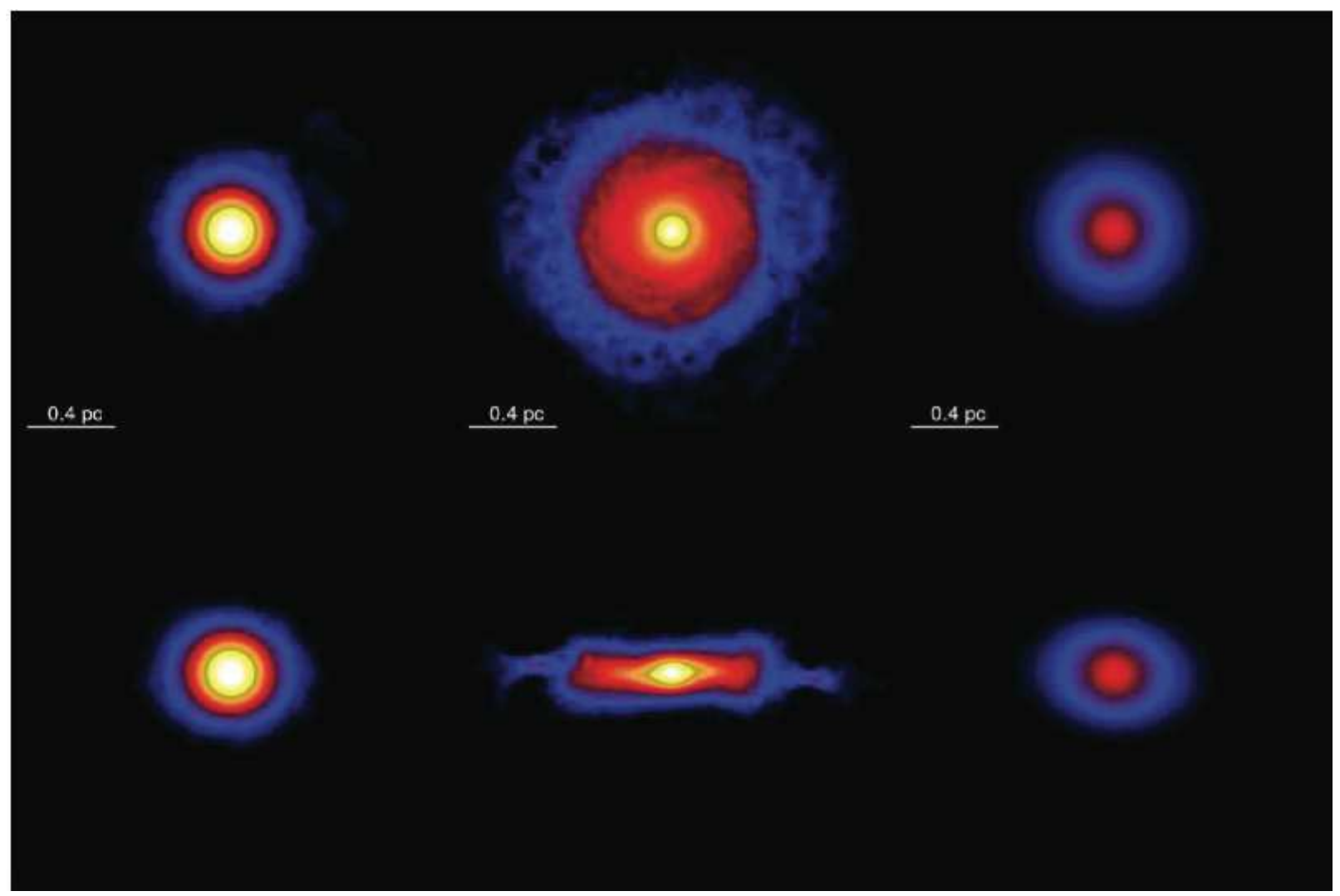

Figure 2. The structure of the final supermassive cloud at pc scales in the MA10 simulations adopting an effective EOS. We show the color coded density maps for the face-on (top, along the angular momentum vector) and edge-on (bottom, perpendicular to the angular momentum vector) views of the cloud after $10^{5} \mathrm{yr}$ from the end of the galaxy merger. From left to right, the end states for equal-mass mergers with galaxies of of different mass are shown (see section 4.1). Note the lowest mass case does not produce a gravitationally bound cloud, while the other two do. The reference case, for a virial mass of $10^{12} M_{\odot}$, is shown on the left. The intermediate mass case gives rise to a rapidly rotating cloud which an unstable to global non-axisymmetric modes that could continue the inflow to smaller scales (see also section 5).

case studied by the analytical model presented in section 3, where stability was predicted irrespective of cooling. This will imply that an effective EOS approach should suffice, However it needs verification with fully radiative simulations, which will be the subject of the next section.

The additional simulations with galaxies with masses a few times $10^{11} M_{\odot}$ show that in this case even stronger gas inflows can arise (Figure 2). This can be understood in terms of the factors that determine the spiral instability in the nuclear disk, namely its density and temperature. At a few times $10^{11}$ the gas mass in the disk is still of order $10^{10} M_{\odot}$ prominent enough to produce a dense self-gravitating nuclear disk after the galaxy merger, but such a disk is colder and thus even more unstable to spiral modes than is our reference simulation. Therefore our direct collapse mechanism could have produced massive black-hole seeds in galaxies that were more common than the high- $\sigma$ peaks hosting the bright quasars at $z>6$, including the most massive progenitors of our own Milky Way. On the contrary, we find that in merging galaxies with virial masses of only $5 \times 10^{10} M_{\odot}$, similar to the present-day Large Magellanic Cloud, a satellite galaxy of our Milky Way, the inflow is too weak to trigger the gravitational collapse of the central region (Figure 2). The light nuclear disk that forms has a very low gas surface density that cannot support a strong spiral instability.

The trend of strength of the gas inflow versus galaxy mass can be understood as a consequence of how the two critical factors determining the stability of the nuclear disk, effective pressure/temperature (including the turbulent part) and surface density, scale with galaxy mass. The argument goes as follows (in the remainder we will always use the scaling laws introduced by Mo, Mao \& White 1998). Thermal and non-thermal (turbulent) energy content of the disk arising from the merger will be determined by the kinetic energy injected by the shock and then thermalized after the two galactic cores collide (note that because of our choice of a stiff $\operatorname{EOS}(\gamma<7 / 5)$ radiative losses are implicitly small, hence it is conceivable to assume thermalization of the kinetic energy). The energy imparted by the shock is $E_{\text {shock }} \propto M_{v i r} V_{v i r}^{2}$ (where the product of the galaxies' virial mass and virial circular velocity squared measures the orbital kinetic energy lost in the collision, assuming a circular orbit for simplicity), or, since $V_{v i r} \sim M^{1 / 3}$ , we have $E_{\text {shock }} \sim M_{v i r}^{5 / 3}$. On the other hand, the surface density of the nuclear disk, which is proportional to that of the host galaxy merger remnant, scales as $\Sigma \sim M_{v i r} /$ Rvir $^{2} \sim M_{v i r}^{1 / 3}$. The disk angular frequency $\Omega \sim V_{v i r} / R_{v i r}$ does not depend on $M_{v i r}$ since $V_{v i r} \sim R_{v i r}$. Therefore, if we define the effective sound speed in the nuclear disk as $c_{s} * \sim E_{i}^{1 / 2}$ (where $E_{i}$ is the internal energy of the gas, including both the ther- 


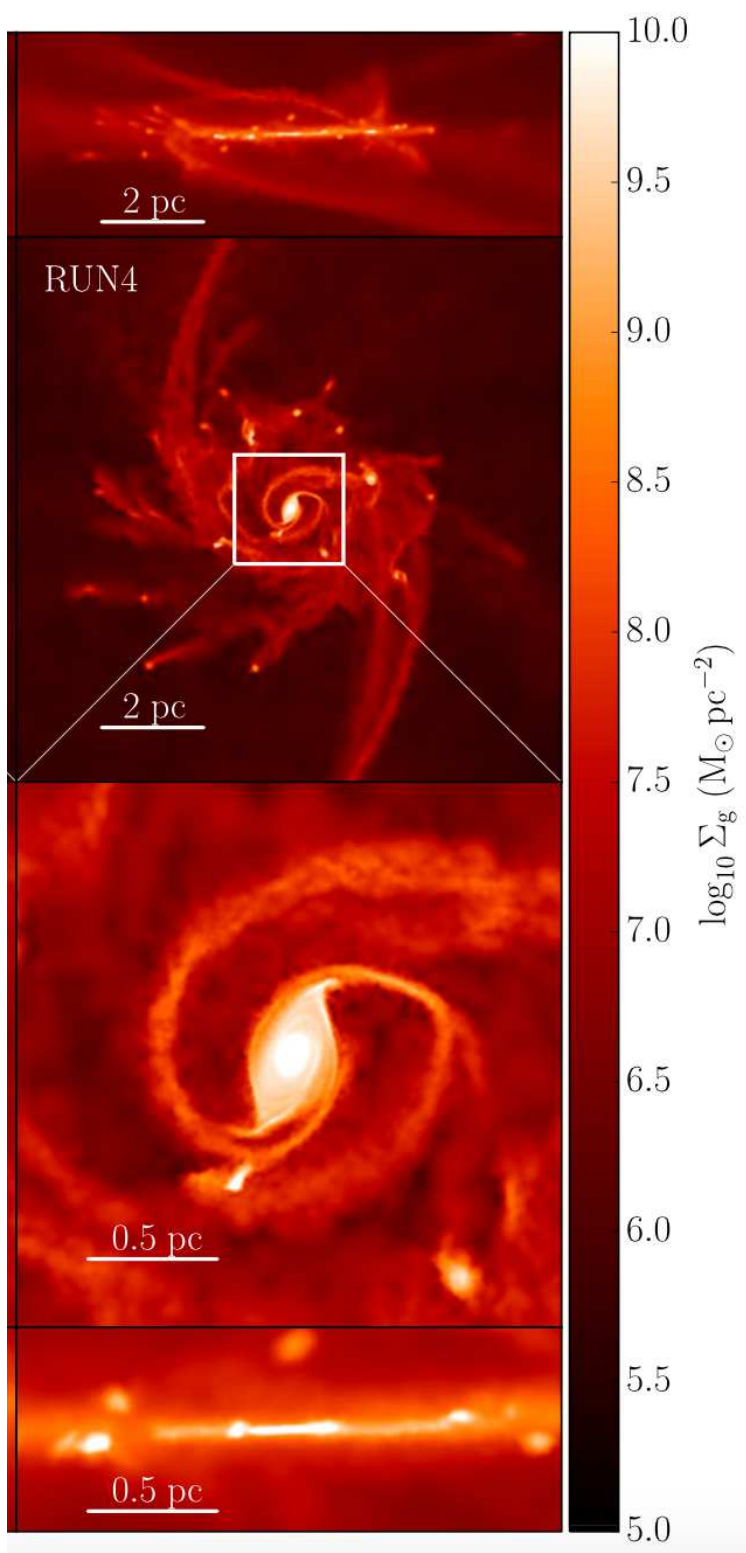

Figure 3. Face-on and edge-on projected gas density maps of the nuclear region of the merger at $t_{0}+5 \mathrm{kyr}\left(t_{0}\right.$ is the time corresponding to the merger of the two central cores.) showing a disklike object with radius 5 pc (first and second panel are edge-on and face-on, respectively), and a compact inner disk-like core less than a parsec in size (third and fourth panels are face-on and edge-on, respectively). We show one particular run from the MA15 paper, RUN4, which employed a modern version of the SPH hydro solver using pressure-energy formulation (GDSPH), thermal and metal diffusion, and a Wedland kernel. As seen in MA15, the structure of the turbulent self-gravitating mini-disk is almost independent on SPH solver and details of the cooling model, which all vary in the different runs presented in MA15.

mal and the kinetic energy component), and assume $E_{i} \sim E_{\text {shock}}$, namely that shock heating sets the level of internal energy of the nuclear disk arising after the collision, the Toomre parameter of the nuclear disk is $Q \sim c_{s} / M_{\text {vir }}^{1 / 2}$. In other words, nuclear disks in higher mass galaxies are increasingly more stable, as the energy imparted by the collision increases more rapidly than the disk self-gravity.

This is the first key result, which explains why the

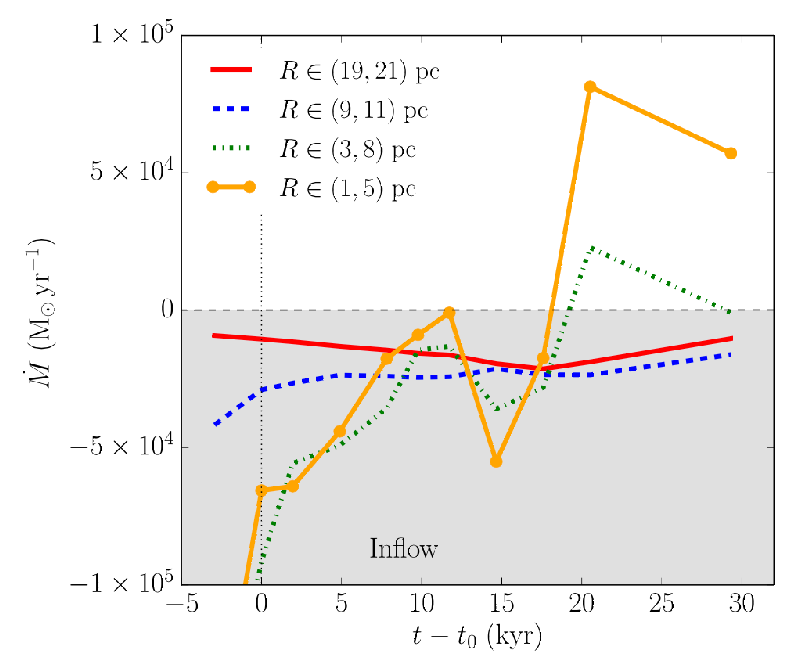

Figure 4. Time evolution of the gas accretion rate at different radii from the center of the merger remnant. The accretion is computed inside cylindrical shells of inner and outer radii marked in the legend and vertical thickness 2 pc. RUN4 from MA15 is used, which is also shown in Figure 3. Adapted from MA15

intermediate mass merger produces a more unstable nuclear disk, and thus a more massive collapsing central gas cloud compared to the reference simulation with higher mass galaxies. However, for increasingly lower mass galaxies the energy imparted by the shock, which scales as $M_{v i r}^{5 / 3}$, will eventually become lower than the initial internal energy of the colliding galaxy cores, so that the surface density of the nuclear disk becomes the most important parameter. In this case we can assume that $c_{s}$ does not depend on $M_{v i r}$ anymore, so that $Q \sim \Sigma^{-1} \sim M_{v i r}^{-1 / 3}$ hence lighter disks are less dense, and therefore more stable. This explains the increased stability of the lowest mass merger that we considered, which results in its inability to undergo central Jeans collapse. The role of shock heating in setting the boundary condition for temperature in the nuclear disk will be confirmed in the radiative simulations of the next section.

As a final cautionary remark, note that the inclusion of radiative cooling, star formation and feedback processes may invalidate the simple scaling arguments employed here. These hold because in EOS simulations the nuclear disk is relatively homogeneous in its temperature structure so that a near steady-state can be assumed. Yet the stifling of the multi-scale instability at low masses should remain as it is generally agreed radiative and kinetic feedback from stars and supernovae becomes a dominant effect eventually, and generates primarily outflows rather than inflows. Indeed, a large number of dwarf galaxy formation simulations (Governato et al. 2010; Onorbe et al. 2015; di Cintio et al. 2014; Wetzel et al. 2015) show that, in galaxies with halo masses only a few tens of billions of solar masses, supernovae-driven gas outflows prevail over merger-driven gas inflows, so that no central gas concentration results and no nuclear disk forms. This is indeed the widely accepted explanation of why dwarf galaxies are bulgeless while massive galaxies always grow a central bulge (Governato et al. 2010). These inferences are based on studying galaxy formation models at relatively late stages $(z<3-4)$, which could cast some doubts on their general validity since at higher redshift gas ac- 


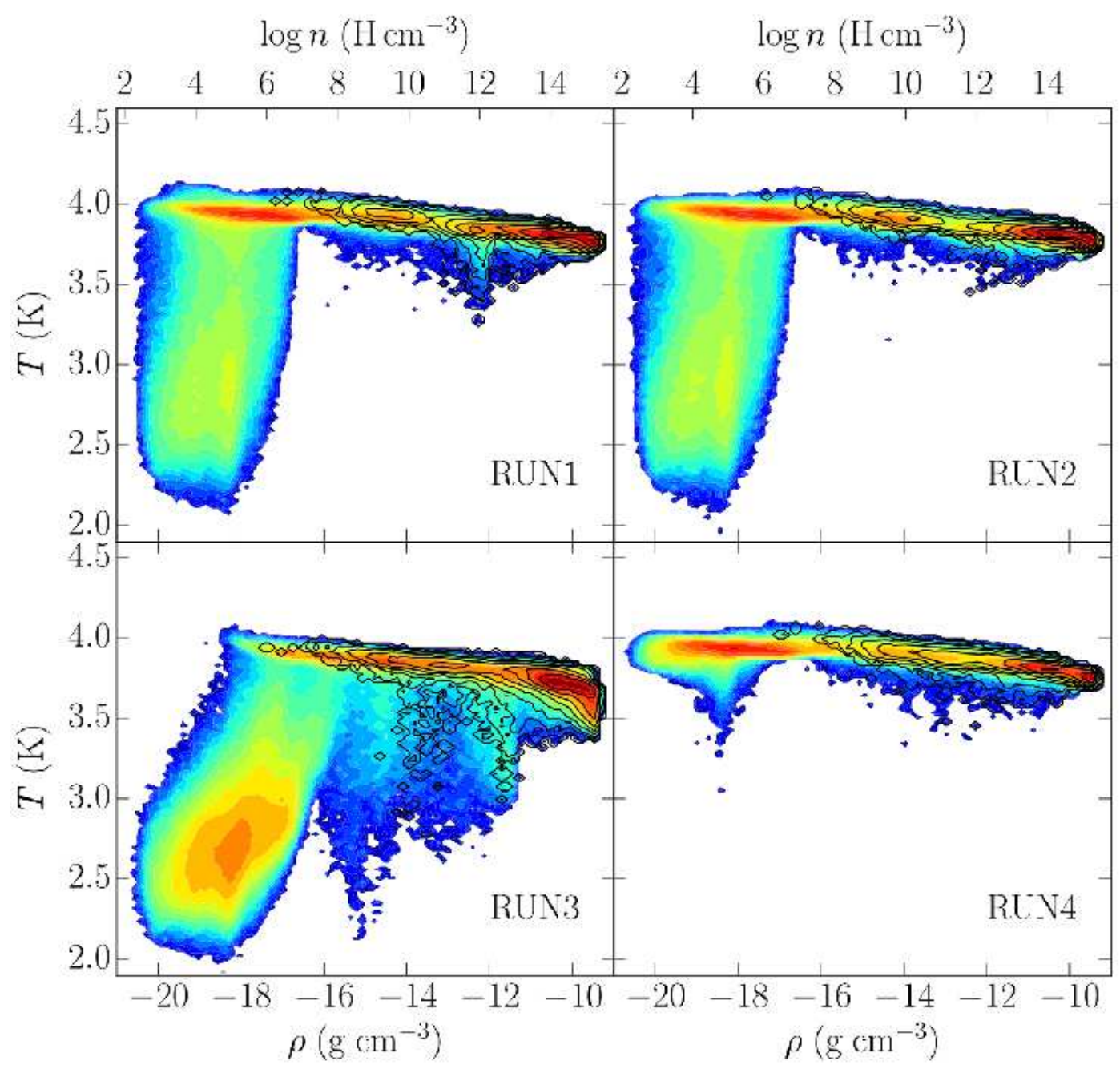

Figure 5. Phase diagrams of the particles within a $50 \mathrm{pc}$ volume at $t=t_{0}+5 \mathrm{kyr}$ for the radiative simulations of MA15. From top-left to bottom-right, we show results for different runs presented in MA15. The two shown at the top are from runs that used vanilla SPH and metal-line cooling but do not include the improved treatment of cooling, heating and self-shielding at high optical depths in the dense core. They differ for details in the implementation of star formation (see MA15). The two shown at the bottom use vanilla SPH but include the effective temperature-density model for high optical depth regions in one case (left), or the modern GDSPH implementation with diffusion terms and Wedland kernel, yet with only standard metal-line cooling using CLOUDY (RUN4, on the right). Black contours are superimposed to show the particles that are located in the inner parsec region, within the inner compact disk. Note that this latter region has almost the same phase diagram in all four runs, consistent with the expectations from the analytical model described in section 3 for which the details of the cooling should not matter rather the behaviour is determined by the gas inflow/accretion rate from large scales. Adapted from MA15.

cretion from the cosmic web should be more prominent and characteristic densities of objects are much higher. However, some recent works studying low mass galaxies $\left(M_{v i r}<10^{11} M_{\odot}\right)$ at $z>5$ in high-resolution hydrodynamical simulations suggest that feedback is also more intense when the specific star formation rate (the star formation rate per unit surface area) is high enough to match that inferred from the data. Feedback then stifles the formation of any coherent dense structure in the inner few tens of parsecs (Fiacconi et al. 2017). While more investigations of the early stages of the formation of low mass galaxies are required, current evidence from galaxy formation simulations strengthens our conclusion that formation of massive black-hole seeds by our flavour of direct collapse would only occur in massive galaxies.

\subsection{Radiative simulations}

\subsection{The radiative cooling model}

Starting from the same initial conditions described above for the more massive models (with halo virial mass of $10^{12} M_{\odot}$ ) we have run four different simulations using GASOLINE2, an updated version of the GASOLINE code (Keller et al. 2014). Individual runs differ in the sub-grid model for radiative cooling as well as for the specific implementation of the SPH equations of hydrodynamics. In all of the runs, we adopt the metal-dependent, optically thin cooling introduced in Shen et al. (2010). It considers tabulated cooling rates in ionization equilibrium, while for $\mathrm{H}$ and $\mathrm{He}$ we directly compute the rates without assuming equilibrium (Wadsley et al. 2004). As in MA10, we assume solar metallicity gas, consistent with observational constraints on the metallicity of the hosts of high-z QSOs (Walter et al. 2004). In one run we include a treatment of the optically thick regime, thereby 
gas above a density of $n_{g}=0.1 \mathrm{~cm}^{-3}$ is on an equilibrium temperature-density relation calibrated on $2 \mathrm{D}$ radiative transfer calculations for a nuclear starburst model. The latter is based on an improved version of the Spaans \& Silk (2000) model (see Roskar et al. (2015) for details). This "thermal balance model accounts for self-shielding effects in a dense ISM and for the heating processes that operate at very high density: (1) cooling by molecular lines and by collisions between dust and molecules with metallicity-dependent opacity effects due to absorption and scattering of photons by dust; (2) IR dust radiation; (3) photoelectric effect on dust; (4) atomic and molecular line trapping in an ISM irradiated by stellar light; and finally (5) heating by cosmic rays. Cooling by $\mathrm{H}_{2}$ is not taken into account in any regime because it is superseded by other molecular cooling channels in a metal-enriched gas, such as CO, which are included in our "metal cooling" CLOUDY module. Furthermore, cooling by fine structure metal lines is actually the dominant process, providing almost two orders of magnitude higher energy loss rate per unit volume compared to molecular cooling at a temperature of a few thousand $K$ even in the high density nuclear region of the merger remnant (see MA15).

The "thermal balance model" assumes the presence of a uniform UV photon radiation field produced by a starburst. Indeed in the large scale merger simulations, when the galaxies are still in the process of coalescing, a starburst with a strength of $\sim 100 M_{\odot} /$ yr takes place in the inner kiloparsec (see MA10). The latter star formation rate thus assumed to determine the stellar UV flux boundary condition in the thermal balance model. While the intensity of the starburst is actually a free parameter, the assumed value is on the low side. Indeed high resdhift starbursts can have easily an order of magnitude higher intensity. This would maintain a higher equilibrium temperature, resulting in even stronger stability of the nuclear compact disk against fragmentation. Note that in this sense a similar notion as in the conventional direct collapse scenario in protogalaxies, namely an external UV flux, would seem to be apply to our model too. However, we will see that, while heating by a starburst is inevitable in our framework, and not an ad-hoc assumption, results will not depend on that or on including self-shielding effects to reduce the cooling rate.

In order to avoid artificial fragmentation we enforce a a pressure floor so that the Jeans mass is resolved locally by at least one resolution element, namely one SPH kernel (see MA15 and Roskar et al. 2015). Most importantly, we do include star formation and blast-wave supernova feedback. The implementation of feedback in the code follows closely Stinson et al. (2006); stars form from gas following a Schmidt law in regions above the density threshold $n_{t h}=10^{4} \mathrm{H} \mathrm{cm}^{-3}$ and below the temperature threshold $T_{t h}=500 \mathrm{~K}$, provided that they are in a convergent flow (measured by the local divergence of the gas velocity field).

We also compare runs with different implementations of SPH that improve on the issue of lack of mixing due to the tensile numerical instability (Agertz et al. 2007), allowing to modelling multi-phase turbulent flows. In particular we compare vanilla-SPH, using a standard density-energy formulation, and GDPSH, which uses a formulation of the hydro force similar to the pressure- energy approach in Hopkins (2013), and includes also a thermal and metal diffusion term (see MA15 and references therein as well as Shen et al. 2010). The results of the selected run shown in Figures 3,4,5 and 6 are those adopting GDSPH.

\subsection{Enhanced inflows and warm loaded nuclear disks}

The main result of these simulations is that, when radiative cooling is included, the multi-scale gas inflows are even more prominent than in the earlier,more idealized simulations with an effective equation of state performed in MA10. This is opposite to the naive expectation that radiative cooling, which here includes metal lines as the gas is assumed by construction to be of solar metallicity, would lead to widespread fragmentation and the disruption of the central inflow. Fragmentation does happen but it is confined only to the outer part of the nuclear compact disk that forms rapidly (Figure 3), in less than $10^{4} \mathrm{yr}$, at the center of the merger remnant. The disk encompasses more than $10^{9} M_{\odot}$ within 1 pc only a few kyr after it begins to assemble (Figure 6 ). Figure 3 shows the state of the system after at two different scales, the smallest being about a parsec (equivalent to 10 resolution lengths). The stronger inflow can be measured by the gas infall rates at various scales (Figure 4), which peak at a value almost an order of magnitude higher than in MA10.This is because efficient cooling renders the inflow nearly isothermal in the nuclear region, removing efficiently the heating generated by shocks. The temperature distribution can be appreciated by inspecting the phase diagrams (Figure 5). As a result a much compact, denser and thinner disk forms relative to the MA10 simulations; its size is below 10 pc as opposed to $100 \mathrm{pc}$ in the previous study. Fragmentation is absent in the inner dense disk core, because the temperature hovers around $5000-6000 \mathrm{~K}$ rather than dropping significantly below that (compare Figure 3 and Figure $5)$. Therefore,somehow unexpectedly the thermal state of the gas at pc scales is very similar to what is obtained in simulations of metal-free protogalaxies illuminated by a dissociating UV source, as in the more conventional direct collapse scenario.

The fact that the inflow leads ultimately to a relatively warm nearly isothermal central collapse in a metalenriched gas is not a trivial outcome. It can be considered the second important result of our work. It is a robust outcome as it is obtained irrespectively of the cooling model adopted in the simulations, for example with or without the thermal balance model, and also does not depend on the specific SPH variant employed (Figure 5).

In order to understand the origin of the nuclear temperature we need to consider the timescales of the key processes involved, and the fact that the system transitions from optically thin to optically thick at different scales as the density increases towards the center. The key underlying point is that there is a competition between radiative cooling and heating by shocks and turbulence. Shock heating dominates in the infalling gas where Mach numbers can be higher than 10 (see MA15), while once gas settles in the dense disk turbulence resulting from gravitational unstabilities in the disk itself becomes the dominant heating source. We verified that, in the absence of any radiative cooling, with the large supersonic infall velocity found in the simulations $(\sim 1000$ 
$\mathrm{km} / \mathrm{s}$ ) the flow would undergo a strong shock yielding a post-shock temperature $>10^{6} \mathrm{~K}$. The infalling gas, though, is still at an optical depth low enough for cooling to take over, bringing the gas to a temperature of few thousand kelvin, but not below that (the shock becomes essentially isothermal). Indeed we verified that the combination of high densities and very high infall velocities in the inner parsec region produces a compressional heating rate that is roughly equal to the cooling rate by fine structure lines around a few $1000 \mathrm{~K}$, while line cooling dominates heating at temperatures above $10^{4} \mathrm{~K}$. These thermal balance arguments explain why the temperature of the disky core hovers around a few thousand $\mathrm{K}$.

Within the dense disk gas cannot cool below a few thousand $\mathrm{K}$ because the optical depth is very large. We will return to the latter timescale in the next section, using the specific conditions found in the simulations. The phase diagrams also show that the "metal cooling" is effective at lowering the temperature in the low density, low Mach number regions far from the core (Figure 5 ). We conclude that the warm temperature maintained by the central disk-like core is a very robust, inevitable product of the supersonic gravitational infall enabled by the larger scale dynamics of a major galaxy merger. The merger essentially provides the ideal case for the loaded disk model presented in the next section.

We also note that no star formation occurs in the warm core simply because the gas never cools enough to meet the temperature condition of our star formation prescription. Sporadic star formation can occur in the densest gas pockets further away from the center, where metalline cooling is effective (Figure 3). The fact that no star formation occurs in the core of the nuclear disk is not only a direct consequence of our star formation prescription, in which a minimum density, a maximum temperature, and the requirement that gas is in a convergent flow locally are set as necessary conditions. Indeed, even if we had set the relevant parameters to very different values, or even if we had used a different prescription based on other relevant properties of the ISM, such as the local Mach number, it would remain true that the gas in the core has a temperature well above the dissociation temperature of molecular hydrogen (2000 K). Since it is almost universally agreed that the presence of a molecular hydrogen phase is necessary for stars to form, it follows that, unless the thermodynamics can be altered significantly, no star formation should occur in the core of the merger remnant. Likewise, the fact that some star formation occurs at the boundary of the disk, inside the dense clumps visible in Figure 3, is expected based on the same argument as the temperature lowers below the dissociation threshold. The dissociation threshold can be considered as a physically motivated temperature threshold that is more conservative than the threshold assumed in our sub-grid model.

The simulations also address the role of turbulent kinetic energy in disk stability, an aspect that is hard to incorporate in an analytical toy-model such as that presented in the next section 4 . At pc scales and below the inner disk gas exhibits a velocity dispersion that is higher than its thermal sound speed. This is the typical signature of a gravoturbulent system, which is expected to settle in a state of marginal stability against fragmentation, namely with a Toomre stability parameter of order unity (see also Choi et al. (2013;2015) and Latif et al. (2013). Indeed this is what is found by measuring the Toomre parameter of the disk in the simulation as $Q=v_{\text {turb }} \kappa / \pi G \Sigma$, namely using the turbulent velocity scale rather than the thermal sound speed as the characteristic velocity scale as it is the largest of the two. Figure 7 shows that most often $Q>1$ except in a few locations where isolated clumps do arise from fragmentation. One exception is the very late stage of the evolution, at $t>10^{4} \mathrm{yr}$, when a second massive clump formed in the outer disk grows significantly in mass and then migrates towards the central disk opening the possibility that two rather just one massive BH seed could be formed (see section 5 and 7 ). Note that, given the short timescales considered here, less than $10^{4} \mathrm{yr}$, these gas clumps cannot form stars but, at most,could collapse below the resolution into a stage similar to a protostellar phase. In doing so they would become emitters of infrared and optical radiation, which will have a negligible effect on the surrounding medium as the effective temperature of such radiation will not be higher than the equilibrium nuclear temperature of a few thousand K.

The heating resulting by gravitational instability is caused by shocks induced by spiral arms, and the turbulent velocity that we measure is nothing else than a proxy for how strong is the non-axisymmetric distortion induced by self-gravity,and hence correlates with the shock strength (see the detailed model of Boley et al. 2010 which performs a relevant analysis for self-gravitating protoplanetary disks where the Mach number of the flow can be directly associated with the overdensity in the spiral arms). This is why we can interpret the relative stability of the disk as the result of heating by gravitoturbulence, which keeps $Q$ generally above unity. Note that when $Q>1$ the cooling timescale becomes a secondary aspect of the physics. The simplest interpretation of our results would then be that gravitoturbulence is the process that appears capable to self-regulate the disk into a marginally stable state characterized by nearly isothermal conditions. Gravitoturbulence will be parametrized by an effective $\alpha$ viscosity in the loaded disk model described in the next section. Furthermore, the temperature remains close to the boundary temperature generated earlier by the supersonic infall. This will justify the the assumption of steady-state of the loaded disk mode introduced in the next section, and is consistent with the notion that the large scale and sub-disk scale inflow rates should be comparable. A difference with the loaded disk model of section 4, though, is that in the simulations the turbulent kinetic energy is not fully thermalized on a disk orbital time, otherwise disk temperature will increase and turbulent velocity will decrease, which is not observed.

There is, however, a possible major caveat in the interpretation so far. Indeed the simulations do no include radiative transfer, hence one may worry that the thermal balance is not correctly computed in the simulations, which could invalidate the whole argument about the self-regulated, gravitoturbulent state. More in general, how can we then assert that what we found should be a generic outcome of gas-rich major mergers at high-z rather than a fluke of the particular assumptions on gas metallicity and the cooling model in the more uncertain 

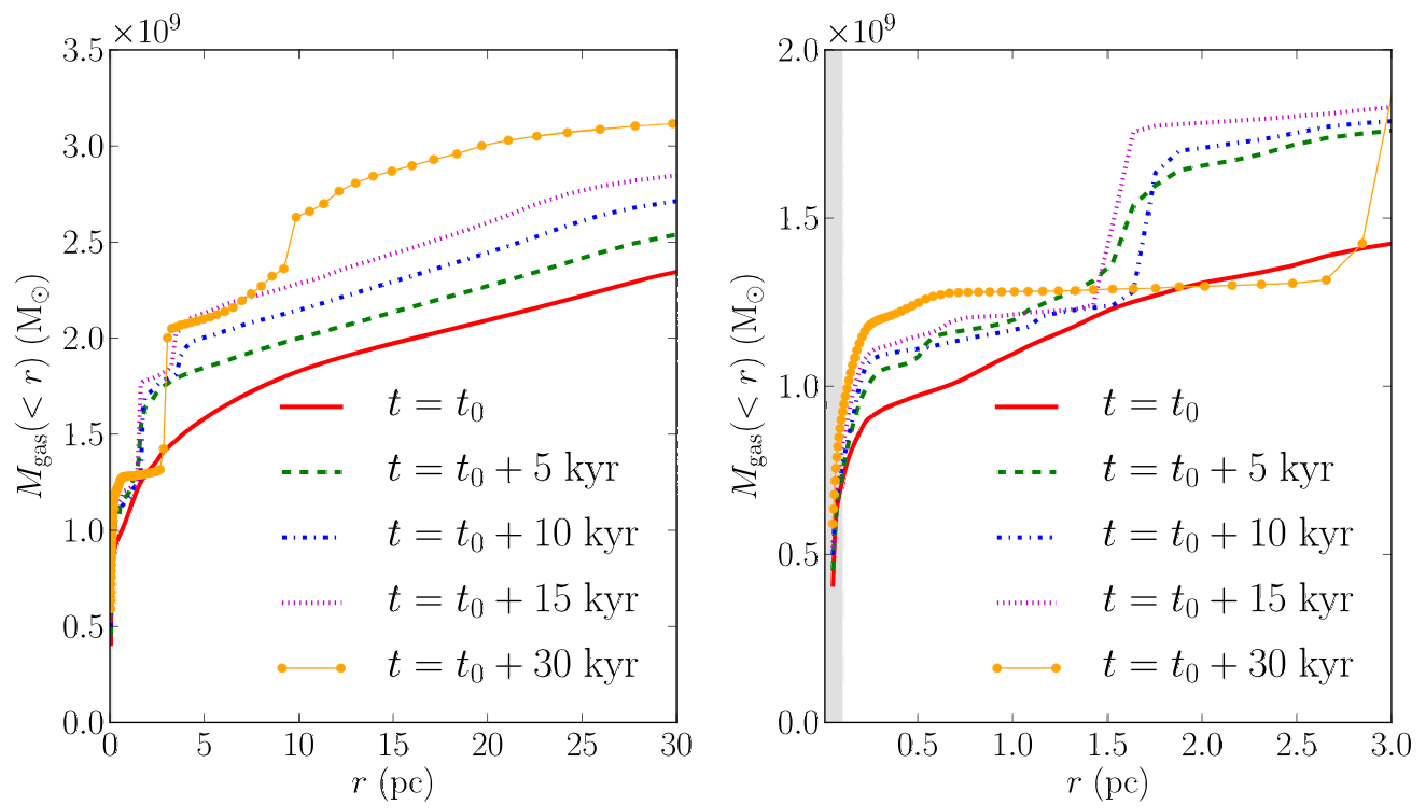

Figure 6. Time evolution of the enclosed gas mass inside $30 \mathrm{pc}$ (left) and inside $3 \mathrm{pc}$ (right) for RUN4. The reference time t0 is the time of the coalescence of the two merging cores (see the text for details). The gray stripe in the right panel highlights the resolution limit set by the gravitational softening. Adapted from MA15.

regime of high optical depths? The answer is found by revisiting the diffusion timescale argument. Let us now consider the cooling timescale in the central disk assuming the diffusion approximation, which is valid in the very high optical depth regime within the central pc. Indeed, the typical optical depth in the disky core within $1 \mathrm{pc}$ is $\tau_{\mathrm{es}} \sim N_{\mathrm{g}} \sigma_{\mathrm{T}} \sim 10^{4}$, where $\sigma_{T} \sim 6.65 \times 10^{25} \mathrm{~cm}^{2}$ is the Thomson scattering cross section and $N_{g} \sim 10^{29} \mathrm{H}$ $\mathrm{cm}^{-2}$ is the mean gas column density within $0.5 \mathrm{pc}$ (corresponding to a surface density $\left.\sim 10^{9} M \odot p c^{-2}\right)$. Note that $N_{g}$ varies by an order of magnitude above and below the quoted value in the very inner region and in the low density gaps between spiral arms and rings, respectively. At the temperature of the core, which is $\sim 3000-5000$ $\mathrm{K}$, the opacity due to electron capture by $\mathrm{H}^{-}$might indeed be up to 10 times higher than that of Thomson scattering for the densities in the inner parsec $\left(\sim 10^{10} \mathrm{~g}\right.$ $\mathrm{cm}-3$ ), making our estimate of the optical depth conservative. Finally, the gas would then cool on the photon diffusion timescale $t_{\text {diff }} \sim H \tau_{\text {es }} / c \sim 3000 \mathrm{yr}$, where $c$ is the speed of light and $H \sim 0.1 R \sim 0.1 \mathrm{pc}$ is the vertical scale height of the disk. This timescale is much longer than the orbital time, which is $\sim 500 \mathrm{yr}$ for $R<1 \mathrm{pc}$, meaning that no fragmentation should occur. Note also that, as we move out to $2-3$ pc the orbital time increases and the optical depth decreases,hence eventually cooling takes over and fragmentation should happen,which is indeed what we observe in the outer disk (Figure 3,5).

A crucial point is that the diffusion timescale calculation just performed is independent on metallicity,just depends on density. A higher metallicity would further increase the true opacity of the gas (namely including all the absorption and scattering processes) but by using $\tau_{e s}$ we have been already very conservative. The conclusion is thus that our result will hold in general as long as we can form such a dense central disk.

\section{THE LOADED DISK MODEL FRAMEWORK AND ITS CONNECTION WITH MERGER-DRIVEN INFLOWS}

Building on the results of the hydrodynamical simulations presented in the previous section, and making use of the concepts of gasdynamics in self-gravitating disks developed in section 2.2, in this section we use simple analytical arguments to sketch a general scenario for the physical evolution of an accreting ultra-dense nuclear disk formed after the major merger of gas-rich galaxies. We will show how the fate of such disk is ignificantly different from that of a nuclear disk inside an isolated low-mass protogalaxy at $z \sim 15-20$.

\subsection{Inflows at sub-galactic scales}

As starting point, we consider the dense nuclear disk with the physical properties described in section 3 for the outcome of the radiative simulations (see 3.3 and 3.4). The characteristic radius of the nuclear disk is then 1-3 pc. While section 3 describes the outcome of simulations carried out in the specific context of major mergers of massive hi-z galaxies, the size of disks in simulations of protogalaxy formation in halos of much lower mass $\left(<10^{10} M_{\odot}\right)$ at $z>15$ are also similar (eg Latif \& Volonteri 2015), as we will recall later in this section. This will allow to generalize our model beyond mergertriggered nuclear disks. The similarity of radii is only coincidental since in one case the centrifugal barrier is connected to the mechanism of angular momentum loss in the merger as gas has to flow inward from kpc-scales in the progenitor galaxies, while in the other case it reflects the fact that the virial radius of high-z mini-halos is by itself a few hundred pc, which translates then in disks a few pc in size since the centrifugal radius is of order a few percent of the virial radius (see section 2). We assume the nuclear mini-disk, either in the merger remnant or in the isolated protogalaxy, to begin in a marginally stable state, $Q \sim 1-1.5$, simply because statistically this is the 
most likely state in which a self-gravitating disk would find itself owing to its tendency towards self-regulation (e.g., Durisen et al. 2007). We recall that our simulations described in section 3 confirm that the nuclear disk is in a marginally unstable state, as shown by the Toomre Q parameter (Figure 7). In order to simplify the algebra involved in the calculations of this section we will further assume $Q=1$. In the remainder, we will show how one can determine a critical surface density $\Sigma_{\text {crit }}$, dependent on the gas infall rate on the disk, above which fragmentation cannot happen because the cooling time becomes too long. The link between surface density, infall rate and cooling time will be provided by the optical depth.

The gas infall rate can then be related to the expected accretion rate at large scales inside the halo, thus closing the loop by allowing to relate quantities associated with galaxy assembly in CDM halos to those describing the pc scale dynamics of nuclear disks. In the case of an isolated protogalaxy the rationale to achieve that was already explained in section 2.1, in particular it follows from eq.2. In the case of a major galaxy merger the interpretation of eq (2) is that this now describes the inflow down to much smaller scales, below the centrifugal radius expected at the scale of the galaxy (kiloparsecs), because the latter has been pushed inward by efficient angular momentum transport triggered by the major merger itself. That is, the centrifugal radius is much smaller than a few percent of $R_{v i r}$, and can only be determined by numerical simulations such as those of section 3 because it is the product of highly nonlinear dynamics. This is ultimately the pc-scale characteristic radius that we have already highlighted. We also remark that we are considering specifically major mergers is important here because it is only in such mergers that angular momentum transport is very efficient (Barnes \& Hernquist 1996; Kazantzidis et al. 2005). For mergers we can still use the scaling laws between virial quantities, which now are those of the merger remnant. For the isolated protogalaxy case a similar approach in spirit was adopted by Lodato \& Natarajan (2006) but the difference here is the notion that accretion rates at multiple scales play a key role in the evolution of the compact disk, in particular deciding whether the disk fragments or not. Note that, in the context of protoplanetary disk formation and evolution, the key role played by accretion has been highlighted by many authors both in numerical simulations and analytical approaches (Boley 2009; Hayfield et al. 2011; Rafikov 2013). We anticipate that the conclusion will be that the new merger-driven model forms central mini-disks that are much more resilient to fragmentation compared to disks in conventional protogalaxies. This will be understood as a consequence of the much higher inflow rates occurring in the merger-driven model which, in turn, naturally follows from the fact that we consider mergers between the most massive galaxies at $z \sim 8-10$ in our model, 2-3 orders of magnitude above the mass of atomic cooling halos considered in the metal-free, dissociated protogalaxy scenario. In other words, the higher inflow rate just reflects that $\dot{M} \propto M_{v i r}$ in eq(2), where $M_{v i r}$ is that of the halo of the merger remnant (which, however, is only a factor of a few different from that of the progenitor galax- ies/halos in a major merger). Such disks, in turn, can sustain coherent secondary inflows to smaller (sub-pc) scales and form a massive precursor of the $\mathrm{BH}$ seed, whose possible nature will be discussed in the next section. We will refer to our model as the loaded disk model.

We can start by computing the angular momentum flux for nearly keplerian self-gravitating disk in steadystate (Rafikov 2013;2015) on parsec scales. Note that, both in the simulations of our merger-driven scenario (MA15), and in the many published simulations of gas inflows in metal-free protogalaxies (e.g., Latif \& Volonteri 2015), the central compact disk that forms has a size of the order of a parsec. As explained in section 2 in the isolated protogalaxy case such a size is expected based on rough conservation of angular momentum of infalling gas into atomic cooling halos with masses in the range $10^{7}-10^{8} M_{\odot}$ (based on the scaling arguments in eg Mo.Mao \& White 1998). Instead, as we explained above, in our merger-driven model the pc-scale size reflects efficient loss of angular momentum caused by the merger dynamics in a much bigger halo, for which the natural size scale would be of order a kiloparsec had angular momentum been conserved (see, e.g., Mayer et al. 2007). These compact disks formed in hydro simulations are not rotating around a central point-like mass as a bona-fide keplerian disk but are extremely centrally concentrated since their early assembly stage. For example, in MA15 we have verified that the disk rotation velocity profile is very close to keplerian down to a fraction of a parsec. The viscous heating originating from gravitoturbulence, which is generated by spiral shocks, is assumed to be the main heating source. This includes also heating from gas-infall as gravitoturbulence will also be sustained by infalling material keeping the disk self-gravitating. External heating sources such as UV-radiation produced by the surrounding star forming region could add to the heating budget hence our calculations in this section should be regarded as conservative for our purpose, that is to show in which conditions the disk will remain warm and avoid fragmentation. The disk is then assumed to be self-luminous, and its state will be determined by the competition between self-generated heat by shocks and gravitoturbulence on one hand, and radiative cooling on the other hand (Kratter \& Lodato 2016). Note that the role of viscous heating in balancing cooling and opposing fragmentation has been studied and advocated also in Latif \& Schleicher (2015), for low-mass protogalaxies at $z>15$. We assume the $\alpha$ effective viscosity framework and, assuming that viscosity is only provided by gravitoturbulence in the disk, $\alpha_{S G}=0.1$ (note the subscript to specify that the source is self-gravity). Indeed $\alpha_{S G}=0.06-0.1$ is the critical threshold for marginally unstable disks. It corresponds to a maximum local gravitational stress (see, eg., Lodato \& Rice 2004). Following Rafikov (2015) we can then write for the angular momentum flux in a self-gravitating disk subject to constant mass transport rate $\dot{M}$ :

$$
3 \pi c_{s}^{2} \Sigma \alpha_{S G}=\dot{M} \Omega
$$

The keplerian disk assumption allows us to replace the epicyclic frequency $\kappa$ with the angular frequency $\Omega$. The thermal sound speed $c_{s}$ will be fixed by as- 
suming a suitable disk temperature. Here $\dot{M}$ is strictly the mass transport through the disk due to spiral density waves and gravitoturbulence. The gas infall from large scales, and its associated infall rate, will be treated as a boundary condition imposed by the large-scale properties of the embedding dark matter halo, which determines the potential well. In steady-state the mass transport rate through the disk and the infall rate onto the disk must match at the disk boundary, hence one can use the latter to set the former. Indeed, the hydrodynamical simulations presented earlier, show that two rates rates, although time-dependent, are relatively commensurate just inside and outside the disk. In this case, we can then replace the $\dot{M}$ of equation 2 with the typical values of accretion rates found in the simulation, which are in the range $1000-5 \times 10^{4} M_{\odot} /$ yr (see Figure 4). To be conservative, here we assume the value of $\dot{M}_{m g}=1000 M_{\odot} /$ yr for the merger case. For the case of the isolated disk, we use the value of the typical infall rates measured in the isolated unstable protogalaxy simulations, $\dot{M}_{i s o} \sim 0.1 M_{\odot} /$ yr (Latif \& Volonteri 2015).

As mentioned above, in equation 3 the angular frequency $\Omega$ is replacing the epicyclic frequency because of the assumption of the disk being keplerian. $\Omega$ is a function of radius, but, for simplicity, we can assume it to be a constant, set by the maximum circular velocity of the host dark matter halo, $V_{\max }$, so that $\Omega=V_{\max } / r$. The maximum circular velocity of a NFW halo occurs typically at 2-3 disk scale lengths, which corresponds to the half-mass radius for a disk with an exponential mass profile, and its value depends on the halo virial mass and the concentration parameter of the halo profile (Bullock et al $2001 b) . V_{\max }$ is typically reached at the halo scale radius $r_{s}$, which roughly also corresponds to the disk half mass radius, and also to $\sim 1 \%$ of the virial radius (see, eg., Mo, Mao \& White 1998). $V_{\max }$ can be from $10 \%$ to $60 \%$ higher than the asymptotic circular velocity $V_{\text {circ }}$ at the virial radius, depending on the halo concentration varying in a reasonable range (5-30, see Figure 1 in Bullock et al. 2001b). Given that we are now focusing on the region dominated by baryons, we will use $V_{\max }$ in what follows. Halo concentration, in turn, depends on $M_{v i r}$ (eg Figures 4-5 in Bullock et al. 2001b). Now, for the merger-driven model we can consider the typical case of a $10^{12} M_{\odot}$ halo, which for a corresponding mean concentration $(c=12)$ yields $V_{\max } \sim 250 \mathrm{~km} / \mathrm{s}$. Note also that changing the halo concentration by a factor of 2 , which occurs over about two decades in halo mass, changes by only $30 \%$ the ratio $V_{\max } / V_{\text {circ }}$ (Bullock et al. 2001b), hence the numbers we are assuming are quite general. As the compact mini-disk is located at $<10$ pc scales, namely at less than $1 \%$ of the virial radius, and the circular velocity profile of massive galaxy mergers steepens towards the center (see, e.g., Guedes et al 2011), this is a lower limit on $V_{\max }$ (this will prove to be onservative for our purpose as it will be clear from the dependencies in the relevant equations below). For isolated protogalaxies halo concentration is higher since the halo mass is 3-4 orders of magnitude lower, hence the concentration will be correspondingly higher. Yet, as the formation redshift is also higher for mini-halos relative to the massive galaxies in the merger case, which implies a lower concentration at fixed halo mass (Bullock et al. 2001b), overall we expect $V_{\max }$ to be again very close to $V_{\text {circ }}$. Solving equation 3 for $\Sigma$, one can find the characteristic surface density that is consistent with a disk transporting mass via gravitoturbulence at a rate in equilibrium with the infall rate from the halo:

$$
\Sigma_{e q}=\frac{\dot{M} V_{\max }}{3 \pi c_{s}^{2} r \alpha_{S G}}
$$

The equation above states the link between large scale and small scale properties of the inflow. We can see that disks with larger mass transport rates embedded in more massive halos yield proportionally a much higher surface density. This is because gas can only flow in, namely no outflows are possible as there are no mechanisms that could generate that. For instance there is no feedback from star formation because star formation can be neglected. The assumption of no star formation, inspired by the the results of section 3 , will shown to be correct $a$ posteriori in order for the model to be self-consistent. It implies that a more massive central disk has to assemble from a larger inflow rate. Note that the radius $r$ of the disk here is a free parameter. It is set by how the specific angular momentum of gas evolves across multiple scales until fluid elements reach centrifugal support. As mentioned at the beginning of the section, we will simply adopt a characteristic radius of $1 \mathrm{pc}$ as we do not model how angular momentum evolves on scales above that of the nuclear disk. We will explore this aspect further in a future update of our analytical model. The same characteristic radius can be assumed in the isolated protogalaxy case (see Latif \& Volonteri 2015; Latif \& Ferrara 2015 and MA15). The similarity of the characteristic radii in the merger and in the isolated protogalaxy case holds despite orders of magnitude differences in the virial masses and radii of the systems considered in the two scenarios because the marked loss of angular momentum in the merger brakes the simple scaling with $R_{v i r}$ for isolated virialized halos (Mo et al. 1998).

From eq. (3) it follows that a disk accreting at a higher rate will be denser, hence will be more optically thick, and thus less prone to fragmentation as cooling will be slower. The higher the temperature, instead, the lower the surface density, as expected since it would correspons to a more prominent role of pressure versus self-gravity in setting the equilibrium in the disk. If we do not place any strong constraint on radiative properties we can posit that disk can cool efficiently via metal lines and molecules, down to (at least) a temperature of 1000 $\mathrm{K}$. This will be used as a reference temperature below, but we will see that results are not highly sensitive to the assumed temperature.

\subsection{Inflows at sub-galactic scales: results of the loaded disk model}

We now proceed to turn the previous equations into a fragmentation threshold dependent on mass loading by exploiting the notion of critical cooling time for fragmentation. The cooling of the disk, if radiative losses dominate over other heat transport mechanisms such as convection (see, e.g, Rafikov 2003), is given by the photon diffusion time, from the disk mid-plane to the surface, which, in line with Section 3, can be expressed by: 


$$
t_{\text {diff }} \sim \frac{H \tau}{c} \sim \frac{N_{g} \sigma_{T} H}{c}
$$

where $N_{g}$ is the column density, $N_{g}=\Sigma / m_{H}, m_{H}$ being the hydrogen mass, and $H$ is the disk scale height. We assume the opacity to be given by electron scattering, which is a lower limit on the total gas opacity, hence will yields a lower limit on the diffusion time. (see also Mayer et al. 2015). By replacing eq(4) into eq(5) using $N_{g}=\Sigma_{e q} / m_{H}$, and by setting $\Sigma=\Sigma_{e q}$, we obtain the following equation for the diffusion time:

$$
t_{d i f f} \sim \Sigma_{e q} / m_{H} \frac{\sigma_{T} H}{c} \sim \frac{\sigma_{T}}{c m_{H}} \frac{\dot{M} V_{\max } H}{3 \pi c_{s}{ }^{2} r \alpha_{S G}}
$$

We recall that we started from the assumption that the disk is in a marginally unstable steady-state maintained by the balance between cooling, heating and gas accretion, and that as a result it supports global nonaxisymmetric modes that render the flow gravitoturbulent. This state can be realized both in an isolated protogalaxy and in a merger remnant since the only thing that changes is the way gas accretion is triggered as well as the value of $\dot{M}$. It simply means that the characteristic surface density of the disk will be different in order to obey the steady-state expressed by eq. (4). Indeed, if we fix $\alpha_{S G}=0.1, r \sim 1 \mathrm{pc}$ and $H=0.3 \mathrm{pc}(\mathrm{H} / \mathrm{r}$ $=0.3$ for a thick, gravitoturbulent disk) , eq(5) can be solved for a given temperature (hence setting the sound speed $c_{s}$ ), a given host halo mass (expressed through the choice of $V_{\max }$ ) and, most importantly, a given accretion/inflow rate $\dot{M}$. Among the latter three parameters $\dot{M}$ is the one with the highest impact since, based on eq. (1), it scales as $M_{v i r}$, which can vary by several orders of magnitude when going from the isolated protogalaxy scenario to our merger-driven scenario, while $V_{\max }$ and $c_{s}$ vary by at most 2 orders of magnitude only across the range of plausible values of temperature and halo mass (recall $V_{\max } \sim M_{\text {vir }}^{1 / 3}$ and $c_{s} \sim \sqrt{T}$ ). Let's now assume $T=1000 \mathrm{~K}, V_{\max } \sim 250 \mathrm{~km} / \mathrm{s}$ and $\dot{M}_{m g}=1000 M_{\odot} / \mathrm{yr}$ for the merger driven model, and $T=1000 \mathrm{~K}, V_{\max }=30 \mathrm{~km} / \mathrm{s}, \dot{M}_{\text {iso }}=0.1 M_{\odot} / \mathrm{yr}$ for the protogalaxy model. For the merger-driven model we then obtain that $t_{\text {diff }} \sim 10^{7} \mathrm{yr}$, while for the other model we obtain $t_{\text {diff }} \sim 10^{3}$ yr. One obtains different orbital times as well, of order $500-1000 \mathrm{yr}$ and $>10^{4} \mathrm{yr}$, respectively, from $T_{\text {orb }} \sim t_{d y n}=1 / \sqrt{G \rho}$ for the average central densities in the two cases, which are, respectively, $\sim 10^{-12}$ $\mathrm{g} / \mathrm{cm}^{3}$ (MA15) and $\sim 10^{-16} \mathrm{~g} / \mathrm{cm}^{3}$ (eg Latif \& Volonteri 2015). The result is thus that for the isolated protogalaxy case the diffusion(cooling) time is shorter than the orbital time, making it a strong case for fragmentation. This argues in favour of finding additional mechanisms to suppress cooling, as it is customarily done in such a scenario by invoking photodissociation of $\mathrm{H} 2$ by $\mathrm{UV}$ radiation and the absence of metals. Instead, the diffusion time is comfortably longer than the orbital time because the high accretion rate in the merger case ensures a high surface density that makes the system optically thick. In this case stability to fragmentation is ensured. A small remark on the temperature, which here is a free parameter, is worthwile. One would be tempted to relate it to the virial temperature of the halo, and thus to $V_{\max }$, in order to eliminate one variable, but this would be incorrect as the disk temperature is the result of cooling and heating processes acting at smaller scales. The thermodynamics in the nucleus of the merger remnants decouples from the simple virial scalings established at the halo level. Moreover, in the merger scenario, if the disk temperature is set as a boundary condition at the level of the large scale flow, it should not change owing to the long cooling times. In the simulations of MA15 it is the shock heating of infalling gas that determines the temperature as a boundary condition (see section 3 ). The latter temperature is $10^{4} \mathrm{~K}$, namely higher than our assumed temperature of $1000 \mathrm{~K}$, but nothing relevant would change in our quantitative arguments so far due to the weak dependence on $c_{s}$ in eq. $(4,6)$.

We thus have shown that, in the simple framework of gravitoturbulent disks in which transport is simply described by an effective $\alpha$ viscosity, the heavily loaded disk in massive halos is by construction resilient to fragmentation without the need of any external heating to suppress radiative cooling. The latter is instead needed in the lighter,mildly loaded central disks arising in isolated low mass protogalaxies. Therefore, in our merger-driven model mass loading replaces external heating sources by maintaining the disk highly self-luminous over long enough timescales, specifically longer than the characteristic orbital time. As anticipated earlier, adding external radiative heating sources, such as from the starburst in the colder, fragmenting gas just outside the nuclear disk, would only strengthen our conclusions. It is also important to remark that we have neglected to explicitly include the compressional heating from the mass loading, which will be more important in the high inflow rate case of the merger-driven model. Hence our conclusions are conservative for multiple reasons in this respect. Note that our analytical model for the heavily loaded disk in principle extends the results of the merger-driven scenario to the general case of high mass infall and transport rate within the disk. We will discuss in the last section how this could be relevant to low tidal field, low spin highly biased regions of the cosmic web, which,according to recent cosmological simulations, indeed are preferential sites of very fast accretion rates onto pre-existing BH seeds (Di Matteo et al. 2017).

The existence of a characteristic surface density for the sustained inflow mode to dominate over the fragmentation mode is reminiscent of the critical column density model developed by Larson (2010) to explain the transition between a regime in which star formation dominates and one in which gas accretion onto a SMBH dominates in lower redshift systems. The Larson model can explain the scaling relation between the mass of SMBHs and the stellar mass of the galactic nucleus or spheroid. In such a model, there exists a gas column density range within which radiative cooling is efficient and gas clouds can become Jeans unstable, so that star formation is the the main outcome, while above a critical column density of $\sim 1 \mathrm{~g} \mathrm{~cm}^{3}$ gas clouds become optically thick to their own cooling radiation, star formation is suppressed, and gas accretes efficiently onto the central black hole. The additional ingredient in our model is the link between optical depth and mass transport rate in the disk, and between 


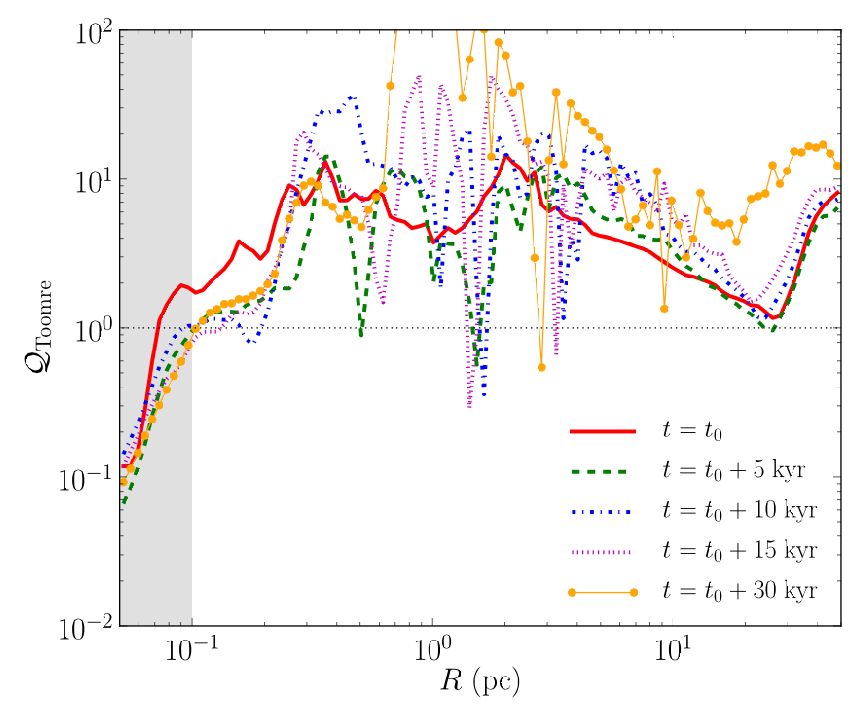

Figure 7. Time evolution of the Toomre parameter profile measured from RUN4 (see the adopted definition of the Toomre parameter in section 4.2, which includes the turbulent velocity). Dips correspond to regions where clumps form from gas fragmentation along spiral arms, predominantly outside the inner parsec region (see Figure 3 for a comparison of the spatial distribution of clumps). The dotted horizontal line marks the $Q=1$ threshold for reference. The gray band highlights the resolution limit given by the gravitational softening. Adapted from MA15.

that and the infall rate from the dark matter halo.

We recall the that the standard $\alpha$ disk is a steady state model, and is only meaningful for a thin disk in which viscous transport is described in a completely local way (Lodato \& Rice 2004;2005). Note that, although it has been often used in the literature, this modelling framework is clearly very approximate in our case as the circumnuclear disks forming in protogalaxies are thick and loaded by accretion of infalling material, and also not strictly keplerian (although they are centrally concentrated as they form inside-out from infalling material). Mass transport by spiral modes is global rather than local in this case since in thick disks non-axisymmetric modes will have large characteristic wavelengths, of order the disk radius, as clearly seen in many simulations. Nevertheless, it has been shown that the global nature of the transport corresponds, empirically, to an effective $\alpha \sim 1$, namely much larger than the nominal 0.1 threshold for fragmentation (see eg Escala et al. 2007). If we had used $\alpha=1$ in the equations above, differences would be minor owing to the linear dependence in $(\mathrm{eq}(5))$. This would yield a somewhat shorter diffusion timescale of $\sim 10^{6} \mathrm{yr}$ in the merger-driven case, which is still orders of magnitude longer than the cooling time (note this is very close to the diffusion time estimate found in MA15 when computing it directly from the properties of the central mini-disk using eq(4)).

Another important caveat is that,although gravitoturbulence is essential in the rationale of the model just described, its direct effect on the dynamics does not appear in the equations. This would be correct if turbulent kinetic energy is rapidly thermalized so that it can be simply absorbed in the choice of $\alpha$ and $c_{s}$. The turbulent velocity in the MA15 simulations, however, remains higher than the thermal sound speed at all times usually,which suggests that, at least in the merger-driven case, it cannot be simply absorbed in other variables. At the same time, persistent turbulent motions will help stabilize further the disk against fragmentation (see section 3.2 ). This could be explicitly treated as a viscous heating term to compare with the diffusion timescale. Latif \& Schleicher (2015) have studied the effect of viscous heating on protogalactic disks, finding that it has a sizeable effect on stabilizing the pc-scale disk. However the effect is smaller than the direct mass loading effect considered here as shown by the fact, in the case of isolated low-mass protogalaxies, they still need some moderate Lyman-Werner flux to avoid fragmentation.

The accretion luminosity can also be added as an extra term but, as shown in MA15, cannot change the basic features of the physical model presented here in the high inflow rate regimes. A change of surface density faster than the cooling time might still bring the system to fragmentation adiabatically (Boley 2009; Hayfield et al. 2011) but if this results from accretion the heating associated should also be taken into account, possibly as viscous heating. These more detailed aspects of the physics of these loaded dense disks warrant future investigations.

\section{FINAL PATHWAYS TO MASSIVE BH SEED FORMATION; THE NOTION OF DARK COLLAPSE}

The models and simulations described in the previous section provide a scenario for the formation of a supermassive baryonic disky core that could later collapse into a massive $\mathrm{BH}$ seed. However they do not address how the formation of the $\mathrm{BH}$ would actually take place. Indeed several different scenarios for the final steps leading, or not, to a massive BH seed can be envisioned, and have been extensively discussed in the literature for the generic direct collapse framework. In the remainder of this section we will discuss the various pathways using quantitative arguments directly related to the results of our simulations. We will argue that our merger-driven scenario lends itself naturally to one particular, intriguing route already proposed in MA15, namely dark collapse. In the latter scenario the compact massive gas core of the mini-disk in the merger remnant could contract further and enter the global General Relativistic (GR) radial instability regime (Hoyle \& Fowler 1963) without transitioning first to a SMS or quasi-star stage. In this case a massive black hole would emerge from direct dynamical collapse of the core, with no intermediate stage. Perhaps the ony aftermath in this case will be gravitational waves due to the non-zero quadrupole introduced by deviations from spherical symmetry (e.g, Saijo \& Hawke 2009), hence the term dark collapse.

Let us begin with considerations on the final outcome of the MA15 simulations, and in particular on the structure and stability of the disky core at the resolution limit. Of course, none of our simulations includes relativistic dynamics hence the discussion in this section will have to be based on simple extrapolations and analytical arguments. Also, even close to the resolution limit, despite the very high densities $\left(\rho \sim 10^{9} \mathrm{~g} / \mathrm{cm}^{3}\right)$ and correspondingly ultra-deep gravitational potential well of the disky core, the system is still well within the newtonian regime until the very end of the simulations, namely after $50 \mathrm{Kyr}$ from the end of the merger (beyond this timesteps were becoming prohibitively small as the central density con- 
tinues to increase, hence we had to stop the calculation). The first question is what would be the next stage of evolution of the compact core if it continues to contract. Would it become a supermassive star (SMS)? With the very high mass and very high accretion rates occurring in our simulation the SMS is somewhat unlikely. Schleicher et al. (2013) show that in order to become an SMS the system must reach a threshold mass dependent on the accretion rate, this being $3.6 \times 10^{8} \dot{m}^{3} M_{\odot}$. Here $\dot{m}$ is the accretion rate in units of solar masses per year. With our rates of $>10^{4} M_{\odot} /$ yr the critical mass is a few orders of magnitude than our core mass of a billion solar masses. The core could then remain in a massive protoSMS stage for long during collapse as the heating from Kelvin-Helmoltz contraction will be high enough to control the collapse.

But could the protoSMS enter the GR radial instability phase at some point as it continues to contract? Let us then consider the conditions for global relativistic radial collapse. In this case, the mass accretion rate does not appear directly in the equations but will still be implicitly instrumental to achieve rapidly the critical mass for collapse in the first place. Numerical GR simulations show that the instability for a rotating fluid configuration (a polytrope with $\gamma=4 / 3$ ) is reached for (1) a compactness threshold $R<640 G M / c^{2}$ and (2) a dimensionless spin parameter $q \equiv c J /\left(G M^{2}\right) \sim 0.97$ (Baumgarte \& Shapiro 1999; Shibata \& Shapiro 2002; Saijo \& Hawke 2009; Reisswig et al. 2013), where $\mathrm{M}$ is the mass and $\mathrm{J}$ is the total angular momentum of the cloud, and $c$ is the speed of light. This has been shown to apply to both uniformly rotating and differentially rotating clouds. The inner disky core in our simulations quickly reaches $\sim 10^{9} M_{\odot}$ within $0.2 \mathrm{pc}$ and it has typically $q \sim 15$. On the other hand, the compactness threshold for $M \sim 10^{9} M_{\odot}$ corresponds to a radius $R \sim 0.03$, which is $\sim 6$ times smaller than the characteristic radius of $0.2 \mathrm{pc}$ found for the final stage of the precursor in the MA15 simulations. Therefore, in the conditions occurring up to the end of the simulations, the disky core would be stable to the GR radial instability. However, our simulations also show that the core is on the verge of a global bar instability (see MA15). Bar modes are barely resolved, hence the instability is artificially suppressed. which we believe is damped by the fact that the wavelength of the most unstable bar mode is barely resolved, hence cannot grow. With increased resolution the instability would grow (Pickett et al. 1996). Once the core eventually becomes bar-unstable, transport angular momentum outwards would become very efficient and cause further contraction, possibly pushing the system closer to the verge of the instability.

An alternative way to discuss susceptibility to global radial collapse is to rely on another result of relativistic simulations, which highlights the importance of angular momentum. Indeed, starting from 2D and 3D rotating polytropic clouds with masses exceeding $10^{6} M_{\odot}$, these simulations show that $T_{\text {rot }} / W \sim 0.01$ or lower is a sufficient condition to bring the cloud to the radial collapse stage under a small initial perturbation (Shibata \& Shapiro 2002). This is of course a phenomenological criterion, and may change with a different EOS, but offers a useful guideline. If we adopt the latter condition, the angular momentum in the inner compact disk has to decrease substantially to enter radial collapse since $T_{\text {rot }} / W \sim 0.1$ at radii $<0.5$ pc. Drawing from the calculations of bar-unstable protostellar clouds, which can apply here since the eventual subsequent contraction will mostly be in the newtonian regime, one expects a decrease of the specific angular momentum, j, by a factor of two over a few dynamical times, (e.g., Pickett et al. 1996), i.e., over $<10^{4} \mathrm{yr}$. At the same time, the mass of the system can grow up to a factor of approximately two, if accretion rates $>10^{4} M_{\odot} / \mathrm{yr}$ are sustained by the bar down to scales $<0.5 \mathrm{pc}$ for $\sim 10^{5} \mathrm{yr}$. Therefore, $T_{\text {rot }} / W$ would decrease by almost an order of magnitude at fixed radius on relatively short timescales (since it scales as $\left.T_{\text {rot }} / W \propto j^{2} M^{-1} R^{-1}\right)$, reaching the critical threshold for radial collapse.

The instability threshold might also be reached first in the inner, denser region. Interestingly, we also measured the radial distribution of angular momentum in the final stage of the disky core evolution in MA15, finding that $T_{\text {rot }} / W$ approaches the conditions for the GR radial instability progressively more towards the inner region. A central small $\mathrm{BH}$ seed might thus arise first and continue to accrete from the envelope, forming essentially a quasi-star (Begelman et al. 2006;2010). However, recent anaytical work incorporating the effects of winds and rotation in quasi-stars find that above a mass of $10^{5} M_{\odot}$ there is no steady-state quasi-star solution (Fiacconi \& Rossi 2016;2017). This basically means that the BH, if formed, will accrete the surrounding envelope on the free-fall timescale (or on a longer viscous timescale if an accretion disk assembles around it), effectively reaching quickly $>10^{8} M_{\odot}$ as in the radial instability scenario. However, in the latter case the $\mathrm{BH}$ seed will shine vigorously as it accretes the entire envelope dynamically, hence there will be an electromagnetic signal, possibly emitting in the James Webb Space Telescope (JWST) bands (Volonteri \& Begelman 2010), this being a major difference from our dark collapse proposal.

In addition to compactness and residual angular momentum there are, however, additional aspects of the physics that play a role in determining the conditions for radial instability. First of all, the protoSMS that could result from the disky core may or may not continue to accrete at very high rates. If high accretion rates continue at small scales, as shown by Ferrara et al. 2013, accretion generates heating that can oppose the contraction, and perhaps push the system away from the GR instability regime at any radius. Hence even if collapse continues it is not guaranteed to end with direct black hole formation. Second, the detailes of thermodynamics cease to matter only when the system is already in the GR instability regime, but until then they can have an impact on the mass growth and in defining the actual threshold for the instability. This means means that one should at least consider the role of the adiabatic index, even remaining in the simple framework of a polytropic equation of state. Numerical GR simulations by Montero et al. 2012, which assume a polytropic protoSMS as an initial configuration, but turn on nuclear burning from the beginning of their calculation, find that metallicity is important below $10^{6} M_{\odot}$, potentially causing a phenomenon similar to the pair-instability in SN. This can result in deflagration of the SMS before it can collapse into a $\mathrm{BH}$. 
Of course the latter calculations are for non-accreting objects hence they exaggerate the role of nuclear burning versus heating by accretion and Kelvin-Helmoltz contraction. Namely, the protoSMS may never become an SMS, as we have argued above following the arguments of Schleicher et al. (2013). For non-accreting supermassive polytropes, irrespective on whether the system persists in a protoSMS phase or becomes an SMS, analytical considerations and direct simulations show that the main physical effect opposing collapse is still rotation. Including both rotation and a variable adiabatic index for an accreting massive polytrope, Ferrara et. 2013 are able to derive a critical threshold mass above which the object should become unstable to the GR radial instability (see also Latif \& Ferrara 2016). The direct effect of rotation and adiabatic index is found to be small, and can be encapsulated in scaling factors to determine the threshold mass, so that one can simply adopt the following expression for the maximum mass of a stable protoSMS:

$$
M_{\max } \sim 6 \times 10^{5}\left(\dot{m} / 1 M_{\odot} y r^{-1}\right) M_{\odot}
$$

where the accretion rate is measured is solar masses per year. The above equation can be obtained if hydrostatic equilibrium holds, namely that the object can continuously adjust to equilibrium as it grows. The collapse below the threshold mass is stabilized by the heating from Kelvin-Helmoltz contraction.

Woods et al. (2017) and Haemmerle et al. (2018) also carried out similar calculations for an accreting SMS but using a full stellar evolution code with an adaptive nuclear network and post-newtonian corrections, for accretion rates up to $10 M_{\odot} / y r$. They found that, for the highest accretion rate, the critical mass for the relativistic instability is about $3.3 \times 10^{5} M_{\odot}$, which is significantly lower than what is predicted by the above equation. These authors still use the notion that the SMS can be described as a polytrope and restrict their calculations to metal-free objects, considering the effect of convection on the equilibrium state. Interestingly, they conclude that there should be an upper limit for the mass of the $\mathrm{BH}$ seed formed by the radial GR instability, and that this is close to $10^{6} M_{\odot}$. The reason is that, as the mass accretion rate increases, the SMS collapses earlier, which limits the mass it can gain before turning into a massive $\mathrm{BH}$. Of course this assumes that there is always an SMS stage preceding the GR instability, while in our dark collapse scenario this would not be the case (or, better, the SMS phase could be so short-lived to be irrelevant for the thermodynamical evolution.) Revisiting these models with even higher accretion rates will be interesting, and is currently in progress. Note that for the extreme accretion rates that we measure in the MA15 simulations, exceeding $1000 M_{\odot} / y r$, the above equation would indicate that the system is marginally unstable within the inner pc, which encompassed $\left(>10^{9} M_{\odot}\right)$. The Woods et al. (2017) results would strengthen further the notion that with such high masses our disky core should definitely become GR-unstable, although a more firm result would demand that their calculations are repeated with our much higher inflow rates. As Ferrara et al. 2013 point out, for masses above $10^{8} M_{\odot}$ there is no simple hydrostatic equilibrium solution as the dynamical time becomes shorter than the Kelvin-Helmoltz contraction timescale. We can add that the analytical treatment brakes down also when the accretion timescale becomes shorter the KH timescale, because in that case the object cannot readjust to equilibrium as it gains mass. The whole notion of the polytrope will not be applicable in such conditions.

We conclude that, although a demonstration will require direct GR simulations of the collapse phase and the ability to model thermodynamics realistically until collapse ensues, there are strong indications that our compact disky core may evolve into a radially unstable protoSMS. Therefore the hypothesis of $\mathrm{BH}$ seed formation by dark collapse appears plausible. Without a prior SMS phase, up to $\sim 60-90 \%$ of the progenitor mass can be retained during such collapse (e.g., Saijo \& Hawke 2009; Reisswig et al. 2013), the emerging BH seed would have a mass roughly between $10^{8}$ and $10^{9} M_{\odot}$, namely in the SMBH mass range from the very beginning. They would be born with masses already close to the mass inferred for the SMBHs powering the high-z QSOs.

If we adopt a more cautious approach, and argue that only a small fraction of the mass of the core will reach the GR instability regime, such as implied by the results of models with a prior SMS phase, as in Woods et al. (2017), or involving a quasi-star phase (Volonteri \& Begelman 2010), still even very conservative assumptions highlight that rapid growth to the required large $\mathrm{BH}$ masses to power high-z QSOs is well within reach. To show this, we can follow the arguments in section 1.2. We can assume the target black hole mass is the largest inferred so far for high-z QSOs, $M_{B H}=10^{10} M_{\odot}$, and that $m_{\text {seed }}=2 \times 10^{5} M_{\odot}$, corresponding to $0.1 \%$ of the mass of the disky core in MA15. Furthermore, we choose standard parameters $f_{e d d}=1$, or $=0.1$ and, to compute the characteristic accretion timescale, we adopt a molecular weight per electron for a plasma at zero metallicity with cosmic abundance of hydrogen $(\mathrm{X}=0.75)$ and helium $(\mathrm{Y}=0.25), \mu_{e}=1 /(1-Y / 2)=1.14$, so that $\tau=0.395$ Gyr. Note that metallicity effects are marginal in this calculation because they only have a negligible effect on the value of the molecular weight. With these choices, we obtain $\left.t_{(} M_{B H}\right) \sim 0.45$ Gyr. This is compatible with the lookback time at $z=6-7$. Assuming that the seed black hole can accrete at the Eddington limit $\left(f_{\text {edd }}=1\right)$ is justified by the fact that the hole would accrete the gas belonging to the nuclear disk, which has very high densities, $n_{H} \sim 10^{5}-10^{8}$ atoms $/ \mathrm{cm}^{3}$ at scales below 10 pc.

In summary, there are strong indications that our scenario will lead to a massive $\mathrm{BH}$ seed on a fast track, although the actual mode of formation in the late stages and the mass of the $\mathrm{BH}$ seed are yet to be determined. It remains to be understood if the statistics of the QSO population that should arise, given the properties of the host galaxies, would match the observations. This will be the subject of the next section.

\section{MERGER SEED FORMATION IN A COSMOLOGICAL CONTEXT AND ITS OBSERVATIONAL CONSEQUENCES: A SEMI-ANALYTICAL APPROACH}

To explore how the scenario of direct collapse formation through galaxy mergers fits in a cosmological context, in Bonoli et al. 2014 (B14) we generalized the re- 


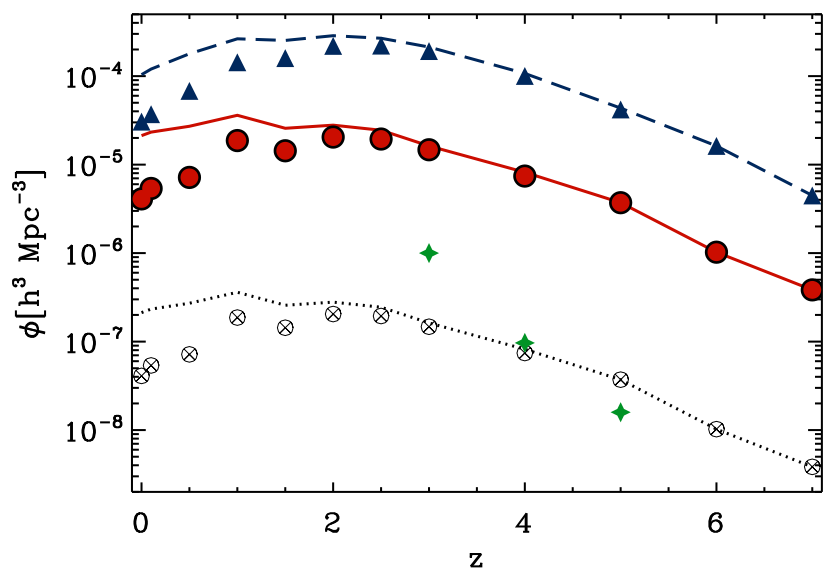

Figure 8. Number density of galaxy major mergers (mass ratio $>0.3$ ) hosted by halos with mass above $10^{11} M \odot$. This is obtained by multiplying the rate of merger events by a $\Delta t$ ("visibility time") of 100Myr (red solid line). The large red dots indicate the number density of events that satisfy the conditions imposed for mergerdriven direct collapse formation. The blue dashed line and the blue triangles are the same as the red line and symbols, but assuming a major merger threshold of 0.1. The black curve and symbols, instead, are the same as the red line and symbols, but assuming a "visibility time" of $1 M y r^{\prime}$. Finally, the green stars indicate the number density of high-redshift quasars calculated by Shen et al. (2007), shown here as reference.

sults of MA10 and conceived a simple analytical model to be incorporated in a framework that follows the evolution of galaxies in a large cosmological volume. In this section, we briefly summarize the results of B14 and discuss future extensions and applications of the model that will allow to make stronger predictions on the frequency of merger-driven seed formation and the testability of our assumptions. Note that the additional aspects of the formation that emerged from the simulations of MA15, in particular the possibility of the dark collapse route, have not been explored yet in the context of this section, although a follow-up model of that in B14, which incorporates the new ingredient, is in progress.

\subsection{A simple generic model for the merger-driven seed formation}

In order study the merger-driven seed model, and its observational consequences, in a cosmological context, we generalized the results of MA10 and devised a simple analytical formulation to be included in a semianalytical galaxy formation model. Semi-analytical models of galaxy formation are powerful tools to study the basic physical processes that drive galaxy evolution, as the global properties of simulated galaxies can be statistically compared with observational information (see, e.g. the seminal works of Kauffmann, White \& Guiderdoni 1993, Cole et al. 1994, Somerville \& Primack 1999). In B14 we made use of $L$-galaxies, the semi-analytic model from the Munich group, in the version presented in Croton et al. 2006 and De Lucia \& Blaizot 2007, and run on the outputs of the N-body Millennium simulation (Springel et al. 2005). With this approach, we were able to follow the cosmological evolution of galaxies residing in halos above $\sim 10^{10} h^{-1} M_{\odot}$, limit set by the mass resolution of the Millennium simulation. In what follows, we explain some details of the model and the main results obtained in B14.
In every new dark matter halo identified in the simulation, a new galaxy is initiated with the baryon fraction corresponding to the assumed cosmological model. After being initalized, galaxies start evolving through gas cooling, star formation, feedback, and other physical processes as explained in Croton et al. 2006 and De Lucia \& Blaizot 2007. In B14 we assume that, when a galaxy is created, a first generation of stars had already been able to create a seed black hole from a PopIII remnant. As we follow the hierarchical evolution of each galaxy, we assume that a galaxy merger event can either lead to the growth of the PopIII remnants via merger and gas accretion, or can lead to the creation of a merger-driven massive seed, if the merger event approximates the initial conditions of the simulations of M10. Specifically, we require (i) that a major merger has taken place (in our default model, we set as threshold for major merger a mass ratio between the stellar and gas components of the two galaxies of $1: 3$ ), (ii) that the merger remnant is hosted by a halo of at least $10^{11} m_{\odot}$, (iii) that the merging galaxies are heavily disk-dominated, with bulge-to-total ratios of, at most, 0.2 and, finally, (iv) the lack of a pre-existing black hole above $10^{6} M_{\odot}$. This last condition is based on energetic arguments, as a pre-existing black hole of already intermediate mass would likely be able, though feedback, to eventually stop the gas inflow needed to form the massive BH precursor. This, of course, would hold in the simple case of isotropic heating; if, instead, radiation is anisotropic, it could still be possible for gas to flow-in at high rates and perhaps lead to Super-Eddington accretion phases on the pre-existing black hole (see Section 7 for a discussion on Super-Eddington accretion).

When the requirements listed above are met, we assume that a massive seed is able to form at the center of the merger remnant, after a rapid quasi-star, SMS or protoSMS phase (see Section 5).

In Figure 8 we show the number density of mergers that satisfy the above conditions, and thus lead to the formation of a massive seed. Note that from the merger rate, which is the information provided by the simulation, we obtain a value for the number density of mergers by assuming a merger "visibility" time of 100 Myr. With this assumption, the number density of all galaxy major mergers, hosted by halos above $10^{11} M_{\odot}$, is shown by the red solid line. The red bullets show instead the number density of the mergers that can lead to the formation of a massive seed, that is, the mergers that satisfy all the other conditions imposed. We see that at high redshift, most mergers could lead to a merger-driven seed. To guide the eye with an order-of-magnitude comparison, we plotted in the same figure the observed number density of optical quasars reported by Shen et al. 2007. While a direct comparison between the observed quasar properties and our black hole population is beyond the scope of this paper, we see that the number of events possibly forming direct collapse seeds is large enough to account for the bright optical quasars. This would hold even assuming a much shorter visibility time of 1 Myr (dotted black curve and black symbols). In the same Figure, the blue dashed line and the blue triangles show the number density of mergers and events of massive seed formation if the threshold for a major merger is decreased from $1: 3$ to $1: 10$ (as used in Volonteri \& Begelman 2010 in 


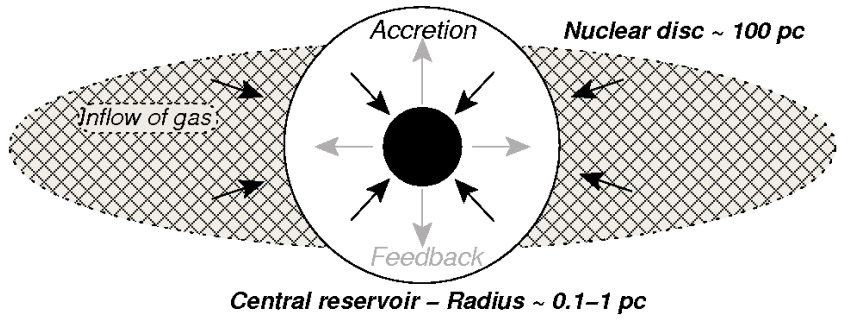

Figure 9. Sketch of the structure around a newly-formed massive seed after a major merges. The seed is surrounded by a gas "reservoir" (white area), which is composed of the remnants of the central massive cloud from which the black hole formed in the first place, and from gas still flowing from the nuclear disk (squared gray region). The growth of the seed stops once its feedback energy balances the binding energy of the central reservoir.

their study of the evolution of quasi-stars). Clearly, the number of events assuming a smaller mass-ratio threshold is much higher, approximately an order of magnitude higher at all redshifts.

After formation, the massive seed is assumed to be able to start accreating at the Eddington rate any gas still available in a gas "reservoir", generated from any residual gas inflow from the circumnuclear disk. The seed stops accreting from this gas reservoir once its feedback is able to unbind the reservoir itself (see Figure 9 for a sketch of the structure surrounding the massive seed soon after formation). In this simple picture, we assumed isotropic and thermal feedback. In the B14 default model, the feedback efficiency is assumed to be $5 \%$ and the central reservoir to have a radius of $1 \mathrm{pc}$.

In Fig. 10 we show, at various redshifts, the resulting black hole mass function, separated depending on the progenitor black hole: the blue solid curve shows the mass function of black holes with a PopIII progenitor, while the different red curves show the mass function of the descendants of merger-driven massive seeds, for different flavours of the model. As mentioned earlier, in our reference merger-seeding model (solid red line), we have assumed a baryonic mass ratio of $1: 3$ for the major merger threshold, a size of 1 pc for the gas "reservoir" from which the newly formed black holes can accrete, and an accretion feedback efficiency of the growing massive seed of $5 \%$. The different red curves in the figure show how sensitive our results are on the choice of these parameters. Compared to the reference model, we get a shift of the mass function to higher masses if we either decrease the physical size of the reservoir (dotted red line) or decrease the efficiency of feedback (dashed red lines) as, in both cases, the newly-formed seed can grow to higher masses before its feedback energy is able to unbind the surrounding gas reservoir. When we instead lower the minimum mass ratio for defining a major merger (dotted-dashed red lines), we see an increase of the normalization of the mass function at all masses, as the number of events that lead to the formation of a massive seed increases by at least an order of magnitude with respect to the reference model. While light seeds descendants are the dominant population at low-redshift, the fraction of black holes with a massive progenitor increases with increasing redshift and mass. Also, we note that at the highest redshift shown in the figure, to reach the masses powering the most luminous quasars (gray line, Willott et al. 2010b), it seems that more frequent
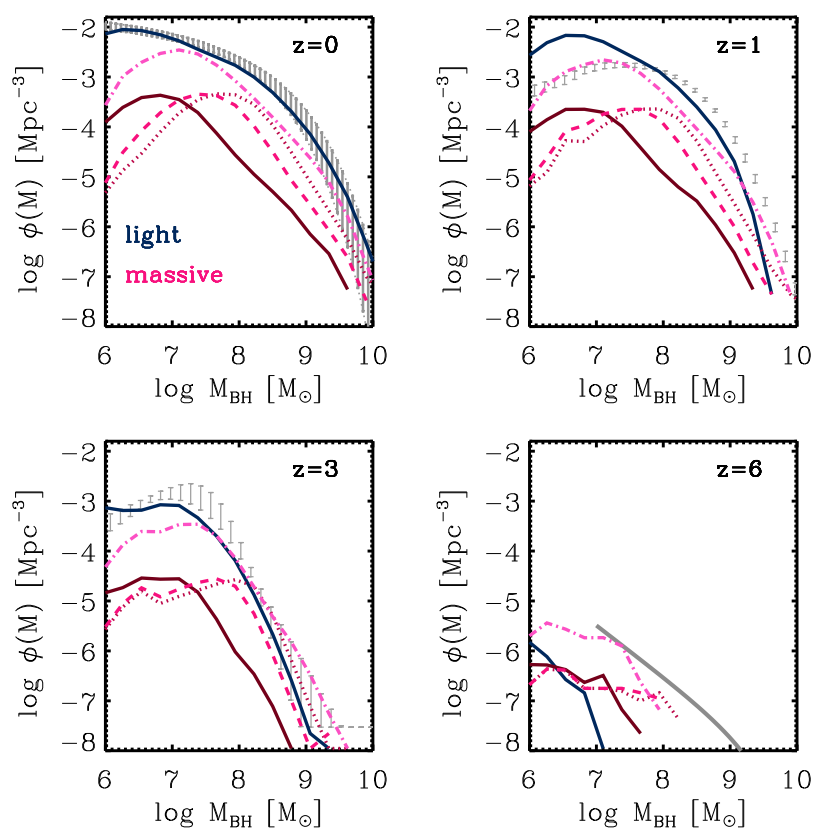

Figure 10. Black hole mass function predicted by the model for the light seed remnants (blue solid curve) and for the merger-driven seeds (red lines), assuming different flavours of the model. The solid line refers to the reference model (reservoir of 1 pc size, $5 \%$ efficiency of feedback coupling, and minimum mass ratio for major mergers of $1: 3$ ). Varying one parameter at a time, for the dotted line we assumed a reservoir of $0.1 \mathrm{pc}$, for the dashed line a feedback efficiency of $1 \%$, and for dot-dashed line a minimum mass ratio of $1: 10$ for major mergers. The gray curves show observational estimates of the black hole mass function (Shankar et al. 2004 at $\mathrm{z}=0$, Merloni \& Heinz 2008 at $\mathrm{z}=1$ and $\mathrm{z}=3$, and Willott et al. $2010 \mathrm{~b}$ at $\mathrm{z}=6$ )

and more massive direct collapse seeds are necessary. We note, however, that the volume probed by the Millennium simulation is not large enough for an exhaustive statistical study of the rare high-z quasars. As further discussed below, we are preparing a new study which include a more comprehensive model for $\mathrm{BH}$ seeds and uses the Millennium-XXL simulation (Angulo et al. 2012), which simulates a cosmological volume 216 times larger.

Finally, one of the advantages of studying a mergerdriven BH seed scenario within the framework of a galaxy formation semi-analytical model, is that not only we can make predictions for the black hole population, but we can also study the properties of the galaxies that host them and their environment. For example, when comparing the merger histories of galaxies that host black holes descendants of massive seeds and of light seeds, we find interesting differences (see Fig. 11): at fixed final black hole mass, while the total number of progenitor galaxies is similar for the light and massive seeds remnants, the typical redshift of the first major merger is very different for the two cases. Galaxies that experienced a major merger early on in their history are much more likely to host a merger-driven seed. This simple result can have important implication in the type of environment in which light and massive seed descendants can be found. Moreover, we also find that, at fixed final black hole mass, massive seeds are more likely hosted by early-type galaxies than light seeds (see Fig. 12). Also, while our reference assumptions for the parame- 

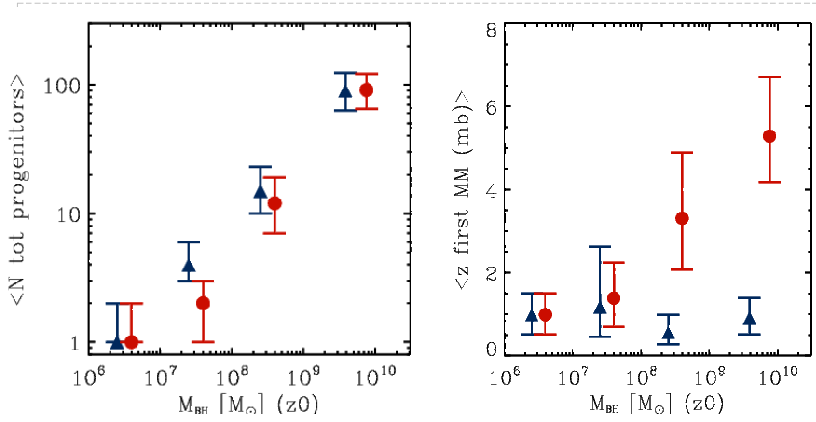

Figure 11. Merger histories of the galaxies hosting light-seeds descendants (blue triangles) and merger-driven seeds (red circles). As a function of final black hole mass, we show, on the left, the median number of progenitors and, on the right, the median redshift of the first major merger. The error bars bracket the $25 \mathrm{th}$ and 75 th percentiles.

ters determining the formation and first growth episode of massive seeds lead to black holes that are consistent with the MBH-MBulge relation, some variations of our reference model, such as a smaller size for the gas reservoir around the new massive seed, lead to a population of black hole that lie above the MBH-MBulge local relation. Such model predictions can be directly tested once a full-census of local black holes is available.

\subsection{A broader look to the black hole seed population}

As discussed above, in B14 we studied the frequency of merger-driven seed formation by incorporating a simple model for the creation of seeds into a galaxy formation framework that tracks the evolution of galaxies in a cosmological context. With this approach, we have been able to study not only the statistics of formation of merger-seeds and properties of their descendants, but also the environment in which these seeds evolve down to the present time. Following-up the results of MA15, an update of B14 is necessary to revise the occurrence of the specific conditions that can lead to a massive seed. In MA15 we briefly investigated the statistics of $z>6$ major mergers in halos above $10^{12} M_{\odot}$, which could potentially sustain strong enough central inflow rates causing a "cold direct collapse" and the direct formation of $\sim 10^{8} M_{\odot}$ seeds. Using the Millennium simulation, we calculated that, by $z \sim 6$, there are $\sim 3 \times 10^{-8} \mathrm{Mpc}^{-3}$ galaxies that live in halos above $10^{12} M_{\odot}$ and already experienced a major merger. This number density is comparable to the number density of known luminous high-z quasars, which is $\sim 10^{-8} \mathrm{Mpc}^{-3}$ (Willott et al. 2010). Work is thus in progress to refine the prescriptions adopted in B14 for the modelling of different seed scenarios, and study in more details the environment of the descendants of the different seed populations. In this work in progress, we are exploiting simultaneously the simulations MillenniumII (Boylan-Kolchin et al. 2009), Millennium (Springel et al. 2005) and Millennium XXL (Angulo et al. 2012), which, combined, offer an extremely large mass dynamic range. Guo et al. 2011, for example, already used the combination of the Millennium and MillenniumII simulations to study the evolution of a wide range of galaxy populations, from dwarfs to large ellipticals. For our purposes, such approach opens the possibility of exploring, at once, the evolution of PopIII seeds, likely developing in more common environments, and of direct collapse
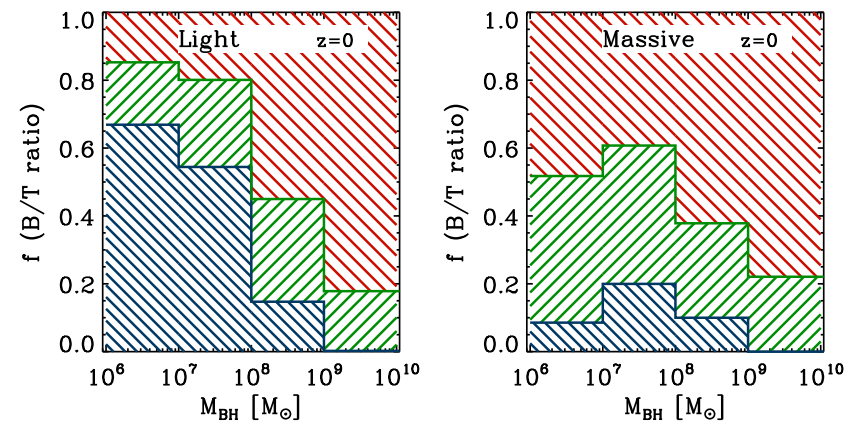

Figure 12. Morphology of BH hosts. Left panel: at fixed final black hole mass, distribution of the morphology ( $\mathrm{B} / \mathrm{T}$ ratio) of the galaxies hosting light seeds descendants. Blue refers to disks or extreme late-types $(\mathrm{B} / \mathrm{T}<0.3)$, green refers to normal spirals $(0.3<\mathrm{B} / \mathrm{T}<0.7)$, while red refers to ellipticals $(\mathrm{B} / \mathrm{T}>0.7)$. Right panel: the same, but for massive seed descendants. While the most massive black holes are hosted primarily by early type galaxies, independent of the progenitor, smaller black holes descendant of massive seeds are generally in more bulge-dominated galaxies than the same black holes descendant of light seeds.

generated in the more rare high-density peaks.

\section{ALTERNATIVE SCENARIOS FOR THE ORIGIN OF BRIGHT HIGH-Z QSOS AND OBSERVATIONAL PROSPECTS}

In this article we have focused on the description of a new scenario for the formation of massive $\mathrm{BH}$ seeds, highlighting the main physical difference with respect to the more standard direct collapse models.

A very different, alternative perspective on the problem of the origin of the extremely massive black holes powering high-z quasars, relies on the notion that the growth rate of initially light seeds, such as those from PopIII stellar remnants, could be much higher than the ones established by the conventional Eddington limit (Madau et al. 2014; Sadowski et al. 2015). Interestingly, the powerful inflows triggered by catastrophic angular momentum loss which are at the base of our mergerdriven direct collapse scenario, can be instrumental to such alternative approach. Indeed, if a light seed could grow at a rate comparable to the inflow rates found in our simulations as well as in our analytical model of loaded disks, clearly the $\mathrm{BH}$ light seed could reach the same mass of our supermassive cloud precursor in similarly short timescales. As discussed in the previous sections, if there is a pre-existing black hole with mass $10^{6} M_{\odot}$ shining at near Eddington luminosity, rapid gas infall could be stifled by radiative feedback. However, if the seed is a few hundred solar masses, its initial accretion luminosity would be very low, and it could grow quickly to at least $10^{6} M_{\odot}$ based on the same argument. Further growth could continue if the radiative efficiency is very low, as in typical Super-Eddington accretion models (e.g., Madau et al. 2014), or if feedback is somehow ineffective at producing enough radiation pressure to overcome the infall of matter. We refer to Johnson \& Haardt (2015) for a recent review on the subject of radiative feedback. Here we just recall the essential aspects of Super-Eddington accretion and the state of research in this topic.

Models of radiatively inefficient accretion discs, such as the SLIM models, in which radiated photons are advected to the black hole instead of diffusing on the photon transport timescale in optically thick gas, provide a con- 
ceptual framework for super-Eddington accretion (Sadowski et al. 2015). Equipped with a SLIM disk solution, semi-analytical models of black hole growth have shown that a light seed of a few hundred solar masses could grow to several billion solar masses in less than a billion years (Madau et al. 2014). In these models, rapid growth essentially arises because of a very low radiative efficiency $\epsilon_{r}<0.01$ which boosts mass growth (see equation Eqn. 1). There are however two potential issues. First of all, the SLIM disk solution is a simple-steady state solution which does not investigate directly the coupling between radiation and advection via turbulence, despite angular momentum transport in accretion disks is believed to be associated with turbulence triggered by processes such as the magnetorotational instability. Stateof-the-art radiation 3D hydrodynamics simulations, such as those by Jiang et al. (2014), which adopt sophisticated radiation transport schemes that naturally allow for anisotropic radiation propagation, find results that differ from the SLIM disk solution. In such simulations, Super-Eddington flows do arise but they are at least an order of magnitude more radiatively efficient than in the SLIM disk solution. However, these simulations have been carried out only for stellar mass black holes and for isolated accretion disks, which have very different flow conditions and environment relative to Pop III seed remnants in highly accreting mini-halos.

Recent $2 \mathrm{D}$ simulations for $\mathrm{BH}$ seeds of $>10^{3} M_{\odot}$, at the high mass end of the Pop III remnants mass distribution (Hosokawa et al. 2013; Hirano et al. 2014) in high density fluid disks, hence more appropriate for the high-z QSOs application, have been published (Inayoshi et al. 2015; Takeo et al. 2018). They have confirmed the existence of Super-Eddington accretion in the equatorial plane while radiation leaks out mostly from the poles. For example, Takeo et al. 2018 find that extremely high accretion rates are possible, in the range 10-100 times higher than the Eddington rate, yet their results do not stem from a fully-self consistent solution of the radiation hydro equations, rather they specify an anisotropic radiation flux a priori, and then apply the diffusion equation within the propagation cones. These flows are of the order of $50 \%$ of the Bondi rate, thus are essentially limited by the larger scale supply rate. However, simulations of mini-halos hosting Pop III remnants show that the gas is warm, diffuse and ionized in such environments, with densities of $1-10 \mathrm{~cm}^{-3}$ (Johnson \& Bromm 2006) rather than the $\sim 10^{5}$ atoms $/ \mathrm{cm}^{3}$ assumed in Takeo et al. 2018. This means the Bondi accretion rate, which is proportional to density, is highly overestimated in Takeo et al. (2018) for the typical case of a BH seed produced by the collapse of a Pop II star. Instead, the conditions for rapid growth via Super-Eddington accretion might be more favourable later on, after the ambient gas has cooled and recombined, raising the characteristic density. This is plausible at $z \sim 10$, and even more so in more massive halos in which gas has collapsed to higher densities to form the central galaxy. These of course would be atomic cooling halos, with virial masses $>10^{8} M_{\odot}$.

Lupi et al. (2016) explored such "late Super-Eddington growth" scenario, addressing it in 3D adaptive mesh refinement (AMR) and lagrangian mesh-less hydrodynamical simulations of circumunclear disks hosted by massive high-z galaxies. By parametrizing the growth of several embedded BHs using an effective (low) radiative efficiency from the SLIM disk model, they do find rapid growth of a light $\mathrm{BH}$ seed $\left(\sim 100 M_{\odot}\right)$ to a million solar, in $<10^{7} \mathrm{yr}$, but cannot follow that further due to the limited volume of the simulations which do not include gas accretion. In such conditions, indeed, growth becomes limited by the gas supply. Moreover, they found that isotropic radiative heating by massive BHs accreting in a spherically symmetric fashion, a popular form of "AGN feedback" adopted in simulations of black hole growth (e.g., Di Matteo et a. 2012; Feng et al. 2014), cannot stifle Super-Eddington accretion. Whether or not circumnuclear disks are common in the nuclei of highredshift galaxies is yet to be seen. A cold self-gravitating disk is instrumental to generate prominent gas inflows via spiral instabilities, which is qualitatively similar to what happens in the merger-driven model. High inflow rates, of the order of the Bondi rate, are also found in simulations of circumnuclear disks (Souza-Lima et al. 2016). What remains to be seen is if such compact disks actually exist in high-z galaxies. In galaxies of typical size and mass at $z \sim 7-8$, this appears unlikely, as stellar and SN feedback drive hot outflows and help to generate a multi-phase, turbulent thick disk (Escala et al 2007; Fiacconi et al. 2017) in which the dynamics is very different from that in Lupi et al. (2016). In such systems accretion is going to be highly anisotropic as it is driven mostly by accreting cosmological filaments (Fiacconi et al. 2017). Locally, the inflow rate could still become Super-Eddington, hence accretion rates could be highly fluctuating. Sustained, episodic Super-Eddington accretion has been proposed as the mode by which even relatively light seeds can grow to very large masses despite feedback effects, possibly explaining also why the fraction of active massive BHs is small at any time despite overall significant growth (Pezzulli et al. 2017a).

It thus appears that growth via Super-Eddington accretion might be more plausible in more massive halos which arise towards $z \sim 10$. Halos as massive as those considered in our merger-driven model at $z \sim 10$ would certainly belong to this category. Mergers would not be necessarily involved but they could aid growth by bringing a large reservoir of gas to parsec scales as the sphere of influence of light Pop III seeds would be quite small. Therefore, the conditions favourable to our scenario would also be very favourable to Super-Eddington accretion. While feedback by the growing black hole would be a difference in the two cases, Lupi et al. (2016) suggests that growth rates are not suppressed because the central black hole seed can augment its mass rapidly by engulfing an entire cluster of stellar and intermediate mass black holes rather than just via gas accretion. New work in progress uses hydrodynamical simulations of massive galaxy formation at $z \sim 10$ with unprecedented resolution, based on re-sampling to sub-pc scales the MassiveBlack runs used in Di Matteo et al. (2012), to study the growth of high-z quasars from already sizeable seeds. Preliminary results suggest that, even before a major merger, tidally interacting massive galaxies at $z>6$ can form a massive ultra-compact disk due to efficient removal of angular momentum by tidal perturbations (Capelo et al. in prep.). This could either give 
a head-start to supermassive cloud precursors of direct collapse black holes, or fuel Super-Eddington accretion. Since resetting the local angular momentum to low values by whatever mechanism seems the key to obtain ultradense central baryonic cores, the local tidal field in the cosmic web might play a significant role in setting the properties of the highly biased hosts of quasars at highz. Recent results from the Blue Tide simulation show indeed a tendency for rapid growth of massive BH seeds to be favoured in regions with a low tidal field, and correspondingly galaxies appear more centrally concentrated in such regions (Di Matteo et al. 2017). Sustained multiscale inflows in such galaxies could be triggered without the need of mergers due to the fact that angular momentum is much lower to begin with. The preliminary results of Capelo et al. (in prep.) also show that a well defined disk is present since $z \sim 10$ in such galaxies, although it is thick and has a relatively high velocity dispersion. Such disks are considerably more gas-rich (with $50 \%$ gas fractions) than the models employed in MA10 and MA15, and globally unstable to large amplitude spiral modes and bar-like modes. Although future analysis will have to address gasdynamics to sub-pc scales, these results suggest that the thin, non-turbulent, stable disk models adopted in the initial conditions of MA10 and MA15 were on the conservative side. Namely, with realistic high-z disks, angular momentum loss could be significant even before the merger is completed.

Observationally, determining the census of very high$\mathrm{z}$ bright quasars at even higher redshift than the ones known today (e.g., in the redshift range 8-15), might be the only way to disentangle between merger-driven direct collapse and Super-Eddington growth of light seeds. The latter regime has to wait to $z<10$, after gas previously ionized in mini-halos has fully recombined, for cooling to happen and gas to be able to feed an eventual BH seed again. Instead, in our merger-driven model, especially in its dark collapse variant, seeds can be so massive from the very start that they could reach $>10^{8} M_{\odot}$ already at $z>10$, thus powering quasars with $L_{X} \sim 10^{43}$ $\mathrm{erg} / \mathrm{s}$ already then. Also, if such population exists already at $z=8-10$, but not earlier, it might favour our scenario against the more conventional scenario of $\mathrm{H}_{2}$-dissociated protogalaxies, as it would be consistent with the notion that sufficiently massive, metal-enriched halos have to appear first for direct collapse to be triggered. The ATHENA X-ray observatory should detect hundreds of AGNs at such very high redshifts, providing very powerful new constraints on competing BH seed formation and/or growth scenarios (Aird et al. 2013).

In addition, studying the evolution of the quasar luminosity function in the redshift range $z \sim 5-15$ will be crucial to test multiple scenarios. Indeed, it is likely that black holes can form and grow via two or more of the channels proposed so far, which hopefully can be distinguished through the different efficiency at which black holes grow. Indeed, if, as it seems currently, there is a dearth of lower luminosity AGNs in the $z \sim 5-10$ range (Treister et al. 2013; Weigel et al. 2015; Pezzulli et al. 2017b), it may imply that lower mass black holes have yet to grow, perhaps from more common, lighter seeds, and that Super-Eddington accretion is not a common route. At the same time, it would imply that the population of bright high-z quasars is special, and likely produced by a direct collapse channel. LISA will provide the only direct way to probe the particular dark collapse idea, since ultra-massive seeds forming in this way will emit a gravitational wave signal during their asymmetric collapse, which can be calculated and is expected to fall in the low frequency band and above the sensitivity curve of the current instrument design (e.g., Saijo \& Hawke 2009).

There have been recent claims that direct collapse black holes have been observed, in particular for CR7, an estimated bright Lyman alpha emitter at $z=6.6$ for which no metal lines could be detected (Sobral et al. 2016; Pacucci et al. 2016). While the presence of a massive black hole powering the emitter is a highly plausible hypothesis (Smith et al. 2016), the absence of metal lines in the spectrum, which would be consistent with the conventional notion that direct collapse black holes require metal-free gas, is not constraining the formation process, only the nature of the accreting gas. Therefore, a light seed black hole from Pop III stars, that has subsequently grown by Super-Eddington accretion in relatively pristine gas, is an equally plausible hypothesis. Detecting a similar source at $z>15$ would instead become interesting in that it would start to really favour direct collapse in metal-free gas as Pop III remnants might still be embedded in ionized, low-density gas at those early epochs (see Section 1), in which Super-Eddington accretion is not feasible. Probing the nuclear cold gas distribution of high $-z$ massive galaxies, in a temperature range below $10^{4} \mathrm{~K}$, as in our compact nuclear disk, could provide also an indirect way to test model assumptions, especially to verify if the extremely high densities and supersonic infall velocities arising in our merger scenario did take place in some systems. The combination of powerful instruments probing the very high redshift Universe at all wavelengths, from ALMA to JWST, ATHENA, WFIRST and SKA, holds the promise to shed light on the fascinating subject of massive black hole formation.

The authors are thankful to the Kavli Institute for Theoretical Physics (KITP) at UC Santa Barbara for hospitality during April-June 2017 during the Program "Galaxy-Halo Connection" where the loaded disk model framework was originally conceived and elaborated. They also acknowledge useful and stimulating discussions on merger-driven direct collapse through the years with M. Begelman, A. Ferrara, M. Latif, R. Schneider, Z. Haiman, P. Natarajan, N. Yoshida, M. Colpi, D. Fiacconi, E. Rossi, K. Schawinski, T. Di Matteo, M. Davies, M. Volonteri, P. Capelo, P. Montero, L. Rezzolla, D. Schleicher.

\section{REFERENCES}

Agertz, O., Teyssier, R., \& Moore, B. 2009, MNRAS, 397, L64

Abel, T., Bryan, G. L. \& Norman, M.L., 2002, Science, 295, 93

Ade et al., Plack collaboration 2016, A\&A, 594, 13

Agertz, O., Teyssier, R., \& Moore, B. 2009, MNRAS, 397, L64

Agarwal, B., et al 2012

Alvarez, M. A., Wise, J. H., \& Abel, T. 2009, ApJ, 701, L133

Amaro-Seoane, P., Aoudia, S., Babak, S., et al. 2013, GW Notes, Vol. 6, p. 4-110, 6, 4

Angulo, R., et al. 2012, MNRAS, 426, 2046

Bañados, E., et al. 2018, Nature , 533, 473

Barnes, J.E, \& Hernquist, L. , 1996, ApJ, 471, 115

Baumgarte, T. W., \& Shapiro, S. L. 1999, ApJ, 526, 941

Begelman, M. C., Volonteri, M., \& Rees, M. J. 2006, MNRAS,

370,289 
Begelman, M. C., Rossi, E. M., \& Armitage, P. J. 2008, MNRAS, 387, 1649

Begelman, M. C., \& Shlosman, I. 2009, ApJ, 702, L5

Begelman, M. C. 2010, MNRAS, 402, 673

Begelman, M. C. 2012, ApJ, 749, L3

Behroozi, P. . Wechsler, R.H., \& Conroy, C., 2013, ApJ, 770, 57

Belkus, H., Van Bever, J., \& Vanbeveren, D. 2007, ApJ, 659, 1576

Boley, A. C. 2009, ApJ, 695, L53

Boley, A. C., Hayfield, T., Mayer, L., \& Durisen, R. H. 2010, Icarus, 207, 509

Boylan-Kolchin, M. et al. 2009, MNRAS, 398, 1150

Bromm, V. \& Loeb, A., 2003, ApJ, 596, 34

Bromm, V., \& Larson, R.B., 2004, ARA\&A, 42, 79

Bonoli, S., Mayer, L., \& Callegari, S. 2014, MNRAS, 437, 1576

Brandt, W.N. \& Hasinger, G. 2005, ARA\&A, 43, 827

Bullock, J. S., et al., 2001 ApJ, 555, 240

Bullock, J. S., et al. , 2001b, MNRAS, 321, 559B

Capelo, P. R., Volonteri, M., Dotti, M., et al. 2014, arXiv: 1409.0004

Ceverino, D., Dekel, A., Mandelker, N., et al. 2012, MNRAS, 420, 3490

Choi, J.-H., Shlosman, I., \& Begelman, M. C. 2013, ApJ, 774, 149

Choi, J.-H., Shlosman, I., \& Begelman, M. C. 2015, ApJ, 450, 4411

Christodoulou, D.M., Shlosman, I, \& Tohline, J.E. 1995, ApJ, 443,551

Cole, S., et al. 1994, MNRAS, 271, 718

Croton, D.J., et al. 2006, MNRAS, 365, 11

Davies, M. B., Miller, M. C., \& Bellovary, J. M. 2011, ApJ, 740, L42

Dekel, A., \& Birnboim, Y., 2006, MNRAS, 368, 2

Deng, H., Mayer, L., \& Meru, F., 2017, MNRAS, submitted

Devecchi, B., \& Volonteri, M. 2009, ApJ, 694, 302

Devecchi, B., Volonteri, M., Rossi, E. M., Colpi, M., \& Portegies Zwart, S. 2012, MNRAS, 421, 1465

Debattista, V. P., Mayer, L., Carollo, C. M., et al. 2006, ApJ, 645,209

De Lucia, G., Blaizot, J., 2007, MNRAS, 375, 2

Di Cintio, A., et al. 2014, MNRAS, 437, 451

Di Matteo, T., Springel, V., \& Hernquist, L., 2005, Nature, 433, 604

Di Matteo, Khandai, N, de Graf, C. , Feng, Y., Croft, R.A.C. Lopez, J., \& Springel, V., 2012, ApJ

Di Matteo, T., Croft, R. A.C. , Feng, Y., Waters, D., \& Wilkins, S., 2017, MNRAS, 467, 4243

Dijkstra, M., Haiman, Z., \& Spaans, M. 2006, ApJ, 649, 14

Dijkstra, M., Ferrara, A., \& Mesinger, A. 2014, MNRAS, 442, 2036

Dotan, C., Rossi, E. M., \& Shaviv, N. J. 2011, MNRAS, 417, 3035

Durisen, R. H., \& Tohline, J. E. 1985, Protostars and Planets II, 534

Durisen, R. H., Boss, A. P., Mayer, L., et al. 2007, Protostars and Planets V, 607

Takeo, E., Inayoshi, K., Ohsuga, K., Takahashi, H, \& Mineshige, S., 2018, MNRAS, 476, 673

Escala, A., 2007, ApJ, 671, 1264

Fan, X., Narayanan, V. K., Lupton, R. H., et al. 2001, AJ, 122, 2833

Feng, Y., Di Matteo, T., Croft, R. \& Khandai, N., 2014, MNRAS, 440,1865

Ferrara, A., \& Loeb, A. 2013, MNRAS, 431, 2826

Ferrara, A., \& Latif, M., 2016, PASA, 33, e051

Fiacconi, D., Mayer, L., Roškar, R., \& Colpi, M. 2013, ApJ, 777, L14

Fiacconi, D., Feldmann, R., \& Mayer, L. 2014, arXiv:1410.6818

Fiacconi, D.' \& Rossi, E., 2016, MNRAS, 455, 2

Fiacconi, D. \& Rossi, E., 2017, MNRAS, 464, 2259

Fiacconi, D., Mayer, L, Madau, P.. Lupi, A., Dotti, M. \& Haardt, F., 2017, MNRAS, 467, 4080

Förster Schreiber, N. M., Genzel, R., Newman, S. F., et al. 2014, ApJ, 787, 38

Freitag, M., Gürkan, M. A., \& Rasio, F. A. 2006, MNRAS, 368, 141

Governato, F., Brook, V, Mayer, L., et al., 2010, Nature, 463, 203

Greif, T, Bromm, V., Clar, P.C. et al., 2012, MNRAS, 424, 39

Johnnson, J. \& Haardt, F., 2017, PASA, 33, e007

Gammie, C. F. 2001, ApJ, 553, 174

Georgiev, I. Böker, T, Leigh, N., Lützgendorf, N. \& Neumayer, N. 2016, MNRAS, 457, 2122

Gillessen, S et al. 2017, ApJ, 837, 30

Girichidis, P., Federrath, C., Banerjee, R. \& Klessen, R.S., 2011, MNRAS, 413, 2741

Glebbeek, E., Gaburov, E., de Mink, S. E., Pols, O. R., \& Portegies Zwart, S. F. 2009, A\&A, 497, 255

Guedes, J. Madau, P., Mayer, L. \& Callegari, S., 2011, ApJ, 729, 125

Guo, Q., et al., 2011, MNRAS, 413, 101
Greif, T.H., Glover, S.C.O., Bromm, V. \& Klessen, R.S., 2010, ApJ, 716, 510

Gürkan, M. A., Freitag, M., \& Rasio, F. A. 2004, ApJ, 604, 632

Habouzit, M., Volonteri, M., Latif, M., Dubois, Y., \& Peirani, S., 2016, MNRAS, 463, 529

Haemmerle, L., Woods, T. E., Klessen, R. S., Heger, A., Whalen, D, J., 2018, MNRAS, 474, 2757

Haiman, Z., 2013, ASSL, 396, 293

Hayfield, T., Mayer, L., Wadsley, J., \& Boley, A. C. 2011, MNRAS, 417, 1839

He, W., et al. 2018, PSAJ, 70, 33

Helled, R., Bodenheimer, P., Podolak, M., et al. 2013, arXiv: 1311.1142

Hernquist, L. 1990, ApJ, 356, 359

Hernquist, L. 1993, ApJS, 86, 389

Hirano, S., Hosokawa., T., Yoshida, N., Umeda, H., Omukai, K. Chiaki, G. \& Yorke, H. W. 2014, ApJ, 781, 60

Hoyle, F. \& Fowler, W.A., 1963. MNRẢ, 125, 169

Inayoshi, K., \& Haiman, Z., 2014, 445, 1549

Inayoshi K., Haiman Z., Ostriker J. P., 2016, MNRAS, 459, 3738

Hopkins, P. F. 2013, MNRAS, 428, 2840

Hopkins, P.F., 2015, MNRAS, 450, 53

Hosokawa, T., Omukai, K., \& Yorke, H. W. 2012, ApJ, 756, 93

Hosokawa, T., Yorke, H.W., Inayoshi. K., Omukai, K., \& Yoshida, N., 2013, ApJ, 778, 178

Jiang Y., F., Stone J.' M., Davis S. W., 2014, ApJ, 796, 106

Jogee, S., 2006, Lecture Notes in Physics,Berlin Springer Verlag, 693,143

Johnson, J.L., \& Bromm, V., 2007, MNRAS, 374, 1557

Johnson, J.L., Whalen, D., Agarwal, B., Paardekooper, J.P. \& Kochfar, S., 2014, MNRAS, 445, 686

Kauffmann., G., White, S.D.M., Guideroni, B., 1993, MNRAS, 264,201

Kaufmann, T., Mayer, L., Wadsley, J., Stadel, J., \& Moore, B. 2007, MNRAS, 375, 53

Kazantzidis, S., Mayer, L., Colpi, M., et al. 2005, ApJ, 623, L67

Keller, B. W., Wadsley, J., Benincasa, S. M., \& Couchman,

H. M. P. 2014, MNRAS, 442, 3013

Khochfar, S., \& Burkert, A. 2006, A\&A, 445, 403

Keres, D., Katz, N., Fardal, M., Dave, R., \& Weinberg, D., 2009, MNRAS, 395, 160

Kormendy, J. \& Ho, L.C. 2013, ARA\&, 51, 511

Kratter, C. \& Lodato, G, 2016, ARA\&A, 54, 271

Kritsuk, A. G., Norman, M. L., \& Wagner, R. 2011, ApJ, 727, L20

Krumholz, M. R., Matzner, C. D., \& McKee, C. F. 2006, ApJ, 653,361

Latif, M. A., Schleicher, D. R. G., \& Spaans, M. 2012, A\&A, 540, A101

Latif, M. A., Schleicher, D. R. G., Schmidt, W., \& Niemeyer, J. 2013, MNRAS, 433, 1607

Latif, M.A., \& Schleicher, D. R. G., 2015, A \& A, 578, 118

Latif, M.A. \& Volonteri, M., 2015, MNRAS, 452, 1026

Latif, M. A., Omukai, K., Habouzit, M., Schleicher, D. R. G., Volonteri, M., 2016, 824, 40

Lin, D.N.C. \& Pringle, J.E. 1987, MNRAS, 225, 607

Lodato, G. \& Rice, W.K.M., 2004, MNRAS, 351, 630

Lodato, G., \& Rice, W.K.M., 2005, MNRAS, 358, 1489

Lodato, G., \& Natarajan, P. 2006, MNRAS, 371, 1813

Lupi, A., Colpi, M., Devecchi, B., Galanti, G., \& Volonteri, M. 2014, MNRAS, 442, 3616

Lupi A., Haardt F., Dotti M., Fiacconi D., Mayer L., Madau P., 2016, MNRAS, 456, 2993

Lynden-Bell, D., 1969, Nature, 223, 690

Madau, P., \& Rees, M. J. 2001, ApJ, 551, L27

Madau, P., Haardt, F., \& Dotti, M. 2014, ApJ, 784, L38

Mayer, L., \& Wadsley, J. 2004, MNRAS, 347, 277

Mayer, L., Wadsley, J., Quinn, T., \& Stadel, J. 2005, MNRAS, 363,641

Mayer, L., Kazantzidis, S., Madau. P., Colpi, M., Quinn, T. \& Wadsley, J. 2007, Science, 316, 1874

Mayer, L., Kazantzidis, S., Escala, A., \& Callegari, S. 2010 Nature, 466, 1082

Mayer, L. 2013, Classical and Quantum Gravity, 30, 244008

Mazzucchelli, C., et al. 2017, ApJ, 849, 91

Merloni, A., Heinz, S., 2008, MNRAS, 388, 1011

Meru, F., \& Bate, M.'R. 2011, MNRAS, 410, 559

Meru, F., \& Bate, M. R. 2012, MNRAS, 427, 2022

Milosavljević, M., Bromm, V., Couch, S. M., \& Oh, S. P. 2009 ApJ, 698, 766

Mo, H. J., Mao, S., \& White, S. D. M. 1998, MNRAS, 295, 319

Moody, C. E., Guo, Y., Mandelker, N., et al. 2014, MNRAS, 444, 1389

Montero, P., Janka, H.T. \& Müller, E. 2012, ApJ, 749, 37

Moster, B. P., Naabm T, \& White, S.D.M., 2017, submitted to ApJ, arXiv: 1705.05373

Mortlock, D. J., et al., 2011, Nature, 464, 616 
Navarro, J. F., Frenk, C. S., \& White, S. D. M. 1996, ApJ, 462, 563

Noguchi, M. 1998, Nature, 392, 253

Omukai, K, ApJ, 546, 635

Onorbe, J., et al., 2015, MNRAS, 454, 2092

Pacucci, F,, Pallottini, A., Ferrara, A. \& Gallerani, S., 2017. MNRAS, 468, L77

Padoan, P., Nordlund, A., \& Jones, B.J.T., 1997, MNRAS, 288, 145

Pardo, K., Goulding, A.D., Greene, J. E., et al. , 2016, ApJ, 831, 203

Pelupessy, F. I., Di Matteo, T., \& Ciardi, B. 2007, ApJ, 665, 107

Pezzulli, E, Valiante, R., Orofino, M. C, Schneider, R., Gallerani, S. \& Sbarrato, T., 2017a, MNRAS, 466, 2131

Pezzulli, E. Volonteri, M., Schneider, R. \& Valiante, R., 2017b, MNRAS submitted, 2017arXiv170606592P

Pickett, B. K., Durisen, R. H., \& Davis, G. A. 1996, ApJ, 458, 714

Portegies Zwart, S. F., Baumgardt, H., Hut, P., Makino, J., \& McMillan, S. L. W. 2004, Nature, 428, 724

Press, W. H. \& Schechter P., 1974, ApJ, 187, 425

Rafikov, R., 2005, ApJL, 621, L69

Rafikov, R., 2013, ApJ, 774, 144

Rafikov, R. 2015, ApJ, 804, 62

Regan, J. A., \& Haehnelt, M. G. 2009a, MNRAS, 393, 858

Regan, J. A., \& Haehnelt, M. G. 2009b, MNRAS, 396, 343

Reisswig, C., Ott, C. D., Abdikamalov, E., et al. 2013, Physical Review Letters, 111, 151101

Rice, W. K. M., Armitage, P. J., Bate, M. R., \& Bonnell, I. A. 2003, MNRAS, 339, 1025

Ritchie, B. W., \& Thomas, P. A. 2001, MNRAS, 323, 743

Roškar, R., Mayer, L., Fiacconi, D., et al. 2014, arXiv:1406.4505

Sadowski, A, Narayan, R., Tchekhovskoy, A., Abarca, D. , Zhu, Y \& McKinney, J.C., 2015, MNRAS, 447, 49

Saijo, M., \& Hawke, I. 2009, Phys. Rev. D, 80, 064001

Saitoh, T.R. \& Makino, J. 2013, ApJ, 768, 44

Saijo, M., \& Hawke, I. 2009, Phys. Rev. D, 80, 064001

Saitoh, T.R. \& Makino, J. 2013, ApJ, 768, 44

Salpeter, E.E., 1964, ApJ, 140, 796

Scalo, J., Vázquez-Semadeni, E., Chappell, D., \& Passot, T. 1998, ApJ, 504, 835

Seth, A., et al., 2008, ApJ, 687, 997

Shakura, N.I, \& Sunyaev, R.A. 1973, A\&A, 24, 337

Shankar, F., Salucci, P., Granato, G.L., DeZotti, G., Danese, L. 2004, MNRAS, 354, 1020

Shapiro, S., 2005, ApJ, 620, 59

Shapiro, S., \& Teukolski.S.A., 1987, "Black holes, white dwarfs and neutron stars; The Physics of Compact Objects", ed . Wiley
Schleicher, D. R. G., Palla, F., Ferrara, A., Galli, D., \& Latif, M. 2013, A\&A, 558, A59

Shen, S., Wadsley, J., \& Stinson, G. 2010, MNRAS, 407, 1581

Shen, Y., et al. 2007, AJ, I33, 2222

Shibata, M., \& Shapiro, S. L. 2002, ApJ, 572, L39

Sobral, D., et al., 2015, ApJ, 808, 139

Somerville, R.S., Primack, J.R. 1999, MNRAS, 310, 1087

Spaans, M., \& Silk, J. 2000, ApJ, 538, 115

Springel, V., et al. 2005, Nature, 435, 629

Stahler, S. W. \& Palla, F. 2005, The Formation of Stars, by Steven W. Stahler, Francesco Palla, pp. 865. I

Stinson, G., Seth, A., Katz, N., et al. 2006, MNRAS, 373, 1074

Tacconi, L. J., Genzel, R., Neri, R., Cox, P., Cooper, M. C., Shapiro, K., Bolatto, A., Bouché, N., Bournau

Tanaka, T., \& Haiman, Z. 2009, ApJ, 696, 1798

Timlin, J. D., et al. 2017, arXiv:1712.03128

Toomre, A., 1964. ApJ, 139, 1217

Trakhtenbrot, B., et al. 2015, Science, 349, 168

Treister, E., Schawinski, K., Volonteri, M., \& Natarajan, P. 2013, ApJ, 778, 130

Van Wassenhove, S., Capelo, P. R., Volonteri, M., et al. 2014, MNRAS, 439, 474

Vink, J. S. 2008, New Astron. Rev., 52, 419

Visbal, E., Haiman, Z., \& Bryan, G., 2014, MNRAS, 445, 1056

Volonteri, M., Haardt, F., \& Madau, P. 2003, ApJ, 582, 559

Volonteri, M., \& Rees, M. J. 2006, ApJ, 650, 669

Volonteri, M., \& Begelman, M. C. 2010, MNRAS, 409, 1022

Walter, F., et al., 2004, ApJ, 615, L17

Wadsley, J. W., Stadel, J., \& Quinn, T. 2004, New A, 9, 137

Weigel, A., Schawinski, K., Treister, E., Urrym C.M., Koss, M. \& Trakhtenbrot, B., 2015, MNRAS 448, 3167

Wetzel, A., Hopkins, P. F., Kim, J.H.,, Faucher-Gigure, C.A., Keres, D., \& Quataert, E., 2016,ApJ, 827, L23

White, S.D.M., \& Rees, M.J., 1978, MNRAS, 183, 341

White, S.D.M., \& Frenk, C.S, 1991, MNRAS ,379, 52

Wilkins, S. M., Feng, Y., Di Matteo, T., Croft, R. et al. 2017, MNRAS, 469, 2517

Willott, C., et al., 2010, AJ, 139, 906

Willott, C., et al., 2010, AJ, 140, 546

Wise, J. H., Turk, M. J., \& Abel, T. 2008, ApJ, 682, 745

Woods, T. E., et al., 2017, ApJL, 842, L6 